Pacific

Journal of

Mathematics

MODULAR TRANSFORMATIONS INVOLVING THE MORDELL INTEGRAL IN RAMANUJAN'S LOST NOTEBOOK

Youn-SeO CHOI

Volume 272 No. 1

November 2014 


\title{
MODULAR TRANSFORMATIONS INVOLVING THE MORDELL INTEGRAL IN RAMANUJAN'S LOST NOTEBOOK
}

\author{
YOUN-SEO CHOI
}

For my teacher Bruce C. Berndt on his 75th birthday.

\begin{abstract}
In his "lost notebook" (p. 202 of the 1988 edition), S. Ramanujan recorded modular transformations involving the Mordell integral, $q$-hypergeometric series, and generalized Lambert series. He gave no proofs; here we prove these formulas and use them to derive modular transformations of thirdorder mock theta functions. Mordell's formula, the properties of $q$-hypergeometric series and Appell-Lerch sums play central roles in the proofs.
\end{abstract}

\section{Introduction}

For a complex number $q$ with $|q|<1$, we define the notation

$$
(a ; q)_{\infty}:=\prod_{m=0}^{\infty}\left(1-a q^{m}\right) \quad \text { and } \quad(a ; q)_{n}:=\frac{(a ; q)_{\infty}}{\left(a q^{n} ; q\right)_{\infty}} \text { for any integer } n .
$$

L. J. Mordell [1920; 1933] studied the integral

$$
\int_{-\infty}^{\infty} \frac{e^{a t^{2}+b t}}{e^{c t}+d} d t
$$

where $\Re(a)<0$. This integral appeared in the work of L. Kronecker [1889a; 1889b] and B. Riemann (as described by C. L. Siegel [1932]). However, Mordell was the first to analyze its behavior relative to modular transformations, so we refer to it as the Mordell integral. In [Mordell 1920] he derived the formula

$$
\begin{aligned}
& \int_{-\infty}^{\infty} \frac{e^{\pi i \tau t^{2}-2 \pi x t}}{e^{2 \pi t}-e^{2 \pi i \theta}} d t \\
& \quad=e^{-\pi i\left(\theta^{2} \tau+2 \theta x+2 \theta\right)} \frac{F[(x+\theta \tau) / \tau,-1 / \tau]+i \tau F(x+\theta \tau, \tau)}{\tau \theta_{11}(x+\theta \tau, \tau)} .
\end{aligned}
$$

This work was supported by NRF-2012047647 from the National Research Foundation of Korea. MSC2010: primary 11B65; secondary 11F27, 33D99.

Keywords: Mordell integral, Appell-Lerch sum, Ramanujan. 
where, for $\Im(\tau)>0$ and setting $q=e^{\pi i \tau}$,

$$
\begin{aligned}
i F(x, \tau) & :=\sum_{m=-\infty}^{\infty} \frac{(-1)^{m} q^{m^{2}+m+1 / 4} e^{(2 m+1) \pi i x}}{1+q^{2 m+1}}, \\
i \theta_{11}(x, \tau) & :=\sum_{m=-\infty}^{\infty}(-1)^{m} q^{m^{2}+m+1 / 4} e^{(2 m+1) \pi i x} .
\end{aligned}
$$

To get (1), he mainly used functional equations satisfied by the functions $F(x, \tau)$ and $\theta_{11}(x, \tau)$.

S. Ramanujan studied definite integrals and recorded modular transformations involving the Mordell integral. In his lost notebook [1988, p. 9], he stated two modular transformations involving Mordell integrals and his tenth-order mock theta functions $\phi(q):=\sum_{n=0}^{\infty} q^{n(n+1) / 2} /\left(q ; q^{2}\right)_{n+1}$ and $\psi(q):=\sum_{n=0}^{\infty} q^{(n+1)(n+2) / 2} /\left(q ; q^{2}\right)_{n+1}$ :

$$
\begin{aligned}
\int_{0}^{\infty} \frac{e^{-\pi n x^{2}}}{\cosh \frac{2 \pi x}{\sqrt{5}}+\frac{1+\sqrt{5}}{4}} d x+ & \frac{1}{\sqrt{n}} e^{\frac{\pi}{5 n}} \psi\left(-e^{-\frac{\pi}{n}}\right) \\
& =\sqrt{\frac{5+\sqrt{5}}{2}} e^{-\frac{\pi n}{5}} \phi\left(-e^{-\pi n}\right)-\frac{\sqrt{5}+1}{2 \sqrt{n}} e^{-\frac{\pi}{5 n}} \phi\left(-e^{\frac{\pi}{n}}\right),
\end{aligned}
$$

$$
\begin{aligned}
\int_{0}^{\infty} \frac{e^{-\pi n x^{2}}}{\cosh \frac{2 \pi x}{\sqrt{5}}+\frac{1-\sqrt{5}}{4}} d x+ & \frac{1}{\sqrt{n}} e^{\frac{\pi}{5 n}} \psi\left(-e^{-\frac{\pi}{n}}\right) \\
& =-\sqrt{\frac{5-\sqrt{5}}{2}} e^{\frac{\pi n}{5}} \phi\left(-e^{-\pi n}\right)+\frac{\sqrt{5}-1}{2 \sqrt{n}} e^{-\frac{\pi}{5 n}} \phi\left(-e^{\frac{\pi}{n}}\right) .
\end{aligned}
$$

In [Choi 2002], we proved these equations. In the lost notebook Ramanujan [1988, p. 202] also wrote (without proofs) two equations involving a Mordell integral, hypergeometric series and generalized Lambert series. Namely, for $q_{1}=e^{-\frac{\pi}{3 n}}$ and $q=e^{-3 \pi n}$,

$$
\begin{aligned}
& \frac{2}{\sqrt{3}} \int_{0}^{\infty} \frac{e^{-\frac{\pi n x^{2}}{3}} \cos \pi t x}{e^{\frac{2 \pi x}{3}}+1+e^{-\frac{2 \pi x}{3}}} d x=q^{\frac{1}{18}} \sum_{m=1}^{\infty} \frac{q^{\frac{(2 m-1)^{2}}{6}}}{\left(-e^{\pi t} q^{\frac{1}{3}} ; q^{\frac{2}{3}}\right)_{m}\left(-e^{-\pi t} q^{\frac{1}{3}} ; q^{\frac{2}{3}}\right)_{m}} \\
& +\frac{e^{-\frac{3 \pi t^{2}}{4 n}} q_{1}^{\frac{1}{2}}}{\sqrt{n}} \sum_{m=1}^{\infty} \frac{q_{1}^{\frac{3}{2}(2 m-1)^{2}}}{\left(-e^{\frac{\pi i t}{n}} q_{1}^{3} ; q_{1}^{6}\right)_{m}\left(-e^{-\frac{\pi i t}{n}} q_{1}^{3} ; q_{1}^{6}\right)_{m}} \\
& =\frac{q^{-\frac{1}{36}}}{\left(q^{\frac{2}{3}} ; q^{\frac{2}{3}}\right)_{\infty}}\left\{\sum_{m=1}^{\infty}(-1)^{m+1} q^{\frac{(2 m-1)^{2}}{4}}\left(\frac{1}{1+e^{\pi t} q^{\frac{2 m-1}{3}}}+\frac{1}{1+e^{-\pi t} q^{\frac{2 m-1}{3}}}-1\right)\right. \\
& \left.+\frac{e^{-\frac{3 \pi t^{2}}{4 n}}}{n} \sum_{m=1}^{\infty}(-1)^{m+1} q_{1}^{\frac{9}{4}(2 m-1)^{2}}\left(\frac{1}{1+e^{\frac{\pi i t}{n}} q_{1}^{3(2 m-1)}}+\frac{1}{1+e^{-\frac{\pi i t}{n}} q_{1}^{3(2 m-1)}}-1\right)\right\} \text {. }
\end{aligned}
$$


We prove these equations in this paper. Proving these identities is equivalent to proving the following two theorems.

Theorem 1. For a positive number $n$, set $q=e^{-3 \pi n}$ and $q_{1}=e^{-\frac{\pi}{3 n}}$. For a number $t$ such that $\Re\left(\frac{t}{n}\right) \pm \frac{2}{3} \notin \mathbb{Z}$ and $\Re\left(\frac{t}{n}\right) \pm \frac{4}{3} \notin \mathbb{Z}$, we have

$$
\begin{aligned}
\frac{2}{\sqrt{3}} \int_{0}^{\infty} \frac{e^{-\frac{\pi n x^{2}}{3}} \cos \pi t x}{e^{\frac{2 \pi x}{3}}+1+e^{-\frac{2 \pi x}{3}}} d x & =q^{\frac{1}{18}} \sum_{m=1}^{\infty} \frac{q^{\frac{(2 m-1)^{2}}{6}}}{\left(-e^{\pi t} q^{\frac{1}{3}} ; q^{\frac{2}{3}}\right)_{m}\left(-e^{-\pi t} q^{\frac{1}{3}} ; q^{\frac{2}{3}}\right)_{m}} \\
+ & \frac{e^{-\frac{3 \pi t^{2}}{4 n}} q_{1}^{\frac{1}{2}}}{\sqrt{n}} \sum_{m=1}^{\infty} \frac{q_{1}^{\frac{3}{2}(2 m-1)^{2}}}{\left(-e^{\frac{\pi i t}{n}} q_{1}^{3} ; q_{1}^{6}\right)_{m}\left(-e^{-\frac{\pi i t}{n}} q_{1}^{3} ; q_{1}^{6}\right)_{m}} .
\end{aligned}
$$

Theorem 2. For a positive number $n$, set $q=e^{-3 \pi n}$ and $q_{1}=e^{-\frac{\pi}{3 n}}$. We have

$$
\begin{aligned}
& q^{\frac{1}{18}} \sum_{m=1}^{\infty} \frac{q^{\frac{(2 m-1)^{2}}{6}}}{\left(-e^{\pi t} q^{\frac{1}{3}} ; q^{\frac{2}{3}}\right)_{m}\left(-e^{-\pi t} q^{\frac{1}{3}} ; q^{\frac{2}{3}}\right)_{m}} \\
& \quad+\frac{e^{-\frac{3 \pi t^{2}}{4 n}} q_{1}^{\frac{1}{2}}}{\sqrt{n}} \sum_{m=1}^{\infty} \frac{q_{1}^{\frac{3}{2}(2 m-1)^{2}}}{\left(-e^{\frac{\pi i t}{n}} q_{1}^{3} ; q_{1}^{6}\right)_{m}\left(-e^{-\frac{\pi i t}{n}} q_{1}^{3} ; q_{1}^{6}\right)_{m}} \\
& =\frac{q^{-\frac{1}{36}}}{\left(q^{\frac{2}{3}} ; q^{\frac{2}{3}}\right)_{\infty}}\left\{\sum_{m=1}^{\infty}(-1)^{m+1} q^{\frac{(2 m-1)^{2}}{4}}\left(\frac{1}{1+e^{\pi t} q^{\frac{2 m-1}{3}}}+\frac{1}{1+e^{-\pi t} q^{\frac{2 m-1}{3}}}-1\right)\right. \\
& \left.+\frac{e^{-\frac{3 \pi t^{2}}{4 n}}}{n} \sum_{m=1}^{\infty}(-1)^{m+1} q_{1}^{\frac{9}{4}(2 m-1)^{2}}\left(\frac{1}{1+e^{\frac{\pi i t}{n}} q_{1}^{3(2 m-1)}}+\frac{1}{1+e^{-\frac{\pi i t}{n}} q_{1}^{3(2 m-1)}}-1\right)\right\} .
\end{aligned}
$$

G. E. Andrews [1981] also studied modular transformations consisting of the Mordell integral and the three functions

$$
\begin{gathered}
M_{1}(q):=\sum_{n=-\infty}^{\infty} \frac{q^{2 n^{2}+n}}{1+q^{2 n}}, \quad M_{2}(q):=\sum_{n=-\infty}^{\infty} \frac{q^{2 n^{2}-n}}{1+q^{2 n-1}}, \\
M_{3}(q):=\sum_{n=-\infty}^{\infty} \frac{q^{2 n^{2}+2 n}}{1+q^{2 n+1}} .
\end{gathered}
$$

These functions are related to the classical theta functions $\vartheta_{2}(0, q)$ and $\vartheta_{4}(0, q)$, and the first two of them appear in Ramanujan's lost notebook.

In [Choi 2011], we made the definition

$$
f(\alpha, z ; q):=\sum_{m=0}^{\infty} \frac{q^{m^{2}-3 m} \alpha^{m} z^{2 m}}{(-z ; q)_{m}\left(-\frac{\alpha z}{q} ; q\right)_{m}} .
$$

If we let $\alpha=z=q$, we see that $f(q, q ; q)$ is one of Ramanujan's famous third-order mock theta functions, $f(q)$, from his letter [Berndt and Rankin 1995]. We can 
rewrite the right-hand side of the equation in Theorem 1 in terms of $f(\alpha, z ; q)$, namely,

$$
q^{\frac{2}{9}} f\left(e^{-2 \pi t} q^{\frac{2}{3}}, e^{\pi t} q^{\frac{1}{3}} ; q^{\frac{2}{3}}\right)+\frac{e^{-\frac{3 \pi t^{2}}{4 n}} q_{1}^{2}}{\sqrt{n}} f\left(e^{-\frac{2 \pi i t}{n}} q_{1}^{6}, e^{\frac{\pi i t}{n}} q_{1}^{3} ; q_{1}^{6}\right) .
$$

Ramanujan's equations involve the hypergeometric series

$$
\begin{gathered}
\sum_{m=1}^{\infty} \frac{q^{\frac{(2 m-1)^{2}}{6}}}{\left(-e^{\pi t} q^{\frac{1}{3}} ; q^{\frac{2}{3}}\right)_{m}\left(-e^{-\pi t} q^{\frac{1}{3}} ; q^{\frac{2}{3}}\right)_{m}}, \\
\sum_{m=1}^{\infty} \frac{q_{1}^{\frac{3}{2}(2 m-1)^{2}}}{\left(-e^{\frac{\pi i t}{n}} q_{1}^{3} ; q_{1}^{6}\right)_{m}\left(-e^{-\frac{\pi i t}{n}} q_{1}^{3} ; q_{1}^{6}\right)_{m}} .
\end{gathered}
$$

These are special cases of the function

$$
g_{3}(z, q):=\sum_{m=1}^{\infty} \frac{q^{m(m-1)}}{(z ; q)_{m}\left(z^{-1} q ; q\right)_{m}} .
$$

Andrews and F. G. Garvan [1989] called attention to what they called the "mock theta conjectures", which roughly say that Ramanujan's fifth-order mock theta functions are not, in fact, theta functions. These were proved by D. Hickerson [1988]; though he did not use the function (2) in the proof, he remarked that he could express the conjectures in terms of it. Since then $g_{3}$ and a couple of other so-called universal mock theta functions have acquired a central role in the study of mock theta functions; see the survey by B. Gordon and R. McIntosh [2012] for discussion.

The function $g_{3}$ also satisfies certain modular transformations [Gordon and McIntosh 2012]. For $q=e^{-\alpha}, q_{1}=e^{-\pi^{2} / \alpha}$, and

$$
h_{3}\left(e^{2 \pi i r}, q\right):=\frac{4 \sin ^{2} \pi r}{(q ; q)_{\infty}} \sum_{m=-\infty}^{\infty} \frac{(-1)^{m} q^{\frac{m(3 m+1)}{2}}}{\left(1-e^{2 \pi i r} q^{m}\right)\left(1-e^{-2 \pi i r} q^{m}\right)},
$$

one of the modular transformations satisfied by $g_{3}$ is

$$
\begin{aligned}
q^{\frac{3}{2} r(1-r)-\frac{1}{24}} g_{3}\left(q^{r}, q\right) & =\sqrt{\frac{\pi}{2 \alpha}} \csc \pi r q_{1}^{-\frac{1}{6}} h_{3}\left(e^{2 \pi i r}, q_{1}^{4}\right), \\
& -\sqrt{\frac{3 \alpha}{2 \pi}} \int_{0}^{\infty} e^{-\frac{3}{2} \alpha x^{2}} \frac{\cosh (3 r-1) \alpha x+\cosh (3 r-2) \alpha x}{\cosh \frac{3}{2} \alpha x} d x .
\end{aligned}
$$

With the function $g_{3}(z, q)$, we can rewrite the right-hand side of Ramanujan's first equation (page 60) as

$$
q^{\frac{2}{9}} g_{3}\left(-e^{\pi t} q^{\frac{1}{3}}, q^{\frac{2}{3}}\right)+\frac{e^{-\frac{3 \pi t^{2}}{4 n}} q_{1}^{2}}{\sqrt{n}} g_{3}\left(-e^{\frac{\pi i t}{n}} q_{1}^{3} ; q_{1}^{6}\right) .
$$


Ramanujan listed four third-order mock theta functions $f(q), \phi(q), \psi(q)$, and $\chi(q)$ in his last letter to G. H. Hardy [Berndt and Rankin 1995]. G. N. Watson [1936] later added three further third-order mock theta functions $\omega(q), v(q)$ and $\rho(q)$, and derived modular transformations for the seven third-order mock theta functions using Cauchy's theorem. One of the modular transformations is

$$
q^{-\frac{1}{24}} f(q)=2 \sqrt{\frac{2 \pi}{\alpha}} q_{1}^{\frac{4}{3}} \omega\left(q_{1}^{2}\right)+4 \sqrt{\frac{3 \alpha}{2 \pi}} \int_{0}^{\infty} e^{-\frac{3}{2} \alpha x^{2}} \frac{\sinh \alpha x}{\sinh \frac{3}{2} \alpha x} d x
$$

where $q=e^{-\alpha}$ and $q_{1}=-\pi^{2} / \alpha$. Gordon and McIntosh [2003; 2012] introduced two more third-order mock theta functions $\xi(q)$ and $\rho(q)$ and their modular transformations.

In his thesis, S. Zwegers [2002] studied the normalized Appell-Lerch sum which is defined by

$$
\mu(u, v ; \tau)=\frac{1}{f\left(-e^{2 \pi i v},-e^{2 \pi i \tau-2 \pi i v}\right)} \sum_{m=-\infty}^{\infty} \frac{(-1)^{m} e^{\pi i m(m+1) \tau+2 \pi i m v}}{1-e^{2 \pi i m \tau+2 \pi i u}}
$$

where $u, v \notin \mathbb{Z} \tau+\mathbb{Z}$ and $\tau \in \mathscr{H}$. He showed the symmetry property, the elliptic transformation properties, and the modular transformation properties satisfied by the normalized Appell-Lerch sum. One of the modular transformation properties contains the Mordell integral, namely,

$$
\left(\frac{\tau}{i}\right)^{-\frac{1}{2}} e^{\frac{\pi i(u-v)^{2}}{\tau}} \mu\left(\frac{u}{\tau}, \frac{v}{\tau} ;-\frac{1}{\tau}\right)=-\mu(u, v ; \tau)+\frac{1}{2} \int_{-\infty}^{\infty} \frac{e^{\pi i x^{2} \tau-2 \pi x(u-v)}}{\cosh \pi x} d x .
$$

With these properties, Zwegers explained that $\mu(u, v ; \tau)$ behaves nearly like a Jacobi form of weight $1 / 2$ in two variables.

Recently, B. Chern and R. C. Rhoades [2012] proved the modular transformation

$$
\begin{aligned}
\widetilde{R}(z ; \tau)-\frac{e^{\frac{3 \pi i z^{2}}{\tau}}}{\sqrt{i \tau}} \widetilde{R}\left(\frac{z}{\tau} ;-\frac{1}{\tau}\right)= & e^{-\frac{\pi i \tau}{3}} i \sin 2 \pi z \int_{-\infty}^{\infty} e^{3 \pi i \tau x^{2}-6 \pi z x} \frac{\cosh 2 \pi \tau x}{\cosh \pi x} d x \\
& +e^{-\frac{\pi i \tau}{3}} \cos 2 \pi z \int_{-\infty}^{\infty} e^{3 \pi i \tau x^{2}-6 \pi z x} \frac{\sinh 2 \pi \tau x}{\cosh \pi x} d x
\end{aligned}
$$

where

$$
\widetilde{R}(z ; \tau):=\frac{i e^{\frac{\pi i \tau}{12}}}{2 \sin \pi z} \sum_{m=0}^{\infty} \frac{e^{2 \pi i \tau m^{2}}}{\left(e^{2 \pi i(z+\tau)} ; e^{2 \pi i \tau}\right)_{m}\left(e^{-2 \pi i(z-\tau)} ; e^{2 \pi i \tau}\right)_{m}} .
$$

They employed the results in Zwegers' thesis [2002] to prove this equation. By results in [Garvan 1988], we can rewrite $\widetilde{R}$ in terms of $g_{3}$ : 


$$
\begin{aligned}
& \widetilde{R}(z ; \tau) \\
& \quad=\frac{i e^{\frac{\pi i \tau}{12}}}{2 \sin \pi z}\left(1-e^{2 \pi i z}\right)\left(1+e^{2 \pi i z} \sum_{m=1}^{\infty} \frac{e^{2 \pi i \tau m(m-1)}}{\left(e^{2 \pi i z} ; e^{2 \pi i \tau}\right)_{m}\left(e^{-2 \pi i(z-\tau)} ; e^{2 \pi i \tau}\right)_{m}}\right) \\
& \quad=\frac{i e^{\frac{\pi i \tau}{12}}}{2 \sin \pi z}\left(1-e^{2 \pi i z}\right)\left(1+e^{2 \pi i z} g_{3}\left(e^{2 \pi i z}, e^{2 \pi i \tau}\right)\right) .
\end{aligned}
$$

In their paper, Chern and Rhoades [2012] also discussed and proved two more identities involving the Mordell integral and partial theta functions. In this paper, Ramanujan's theta function $f(a, b)$ is used instead of the Jacobi theta functions. The definition of Ramanujan's theta functions is, for $|a b|<1$,

$$
f(a, b):=\sum_{m=-\infty}^{\infty} a^{m(m+1) / 2} b^{m(m-1) / 2} .
$$

By the Jacobi triple product identity, this equals $(-a ; a b)_{\infty}(-b ; a b)_{\infty}(a b ; a b)_{\infty}$.

In Section 2, we introduce Lemmas 1 and 2. The identities in these lemmas include generalized Lambert series which are the Appell-Lerch sums. The transformation for the Appell-Lerch sum in [Zwegers 2002] plays a central role in the proofs of Lemmas 1 and 2. In Section 3, we prove Theorem 1 twice with distinct methods. We first prove Theorem 1 by using Lemmas 1 and 2, Mordell's formula, the modular transformation for a theta function $\theta_{11}$, and the evaluations of the contour integrals. Secondly, we prove Theorem 1 by proving

$$
\frac{1}{\sqrt{3}} \int_{-\infty}^{\infty} \frac{e^{\frac{\pi i \tau x^{2}}{3}-2 \pi z x}}{e^{\frac{2 \pi x}{3}}+1+e^{-\frac{2 \pi x}{3}}} d x=g(z ; \tau)+\frac{e^{\frac{3 \pi i z^{2}}{\tau}}}{\sqrt{-i \tau}} g\left(-\frac{z}{\tau} ;-\frac{1}{\tau}\right)
$$

where

$$
g(z ; \tau):=\frac{e^{\frac{2 \pi i \tau}{3}}}{\left(e^{2 \pi i \tau} ; e^{2 \pi i \tau}\right)_{\infty}} \sum_{m=-\infty}^{\infty} \frac{(-1)^{m} e^{3 \pi i \tau m(m+1)}}{1+e^{2 \pi i z+2 \pi i \tau\left(m+\frac{1}{2}\right)}} .
$$

To prove the equation above, we discuss the elliptic transformation properties of $g(z ; \tau)$, evaluate the contour integrals, and employ Liouville's theorem. In Section 4, we prove Theorem 2 by using Equation (6) and some results in the first proof of Theorem 1. In Section 5, with Theorem 1, we derive modular transformations for third-order mock theta functions which are similar to the modular transformations for tenth-order mock theta functions in the lost notebook [1988, p. 9].

\section{Lemmas}

To prove Theorems 1 and 2, we require the following lemmas. 
Lemma 1. For a complex number $q$ with $|q|<1$, we have

$$
\begin{aligned}
& \sum_{m=1}^{\infty} \frac{q^{\frac{2 m(m-1)}{3}}}{\left(-t q^{\frac{1}{3}} ; q^{\frac{2}{3}}\right)_{m}\left(-t^{-1} q^{\frac{1}{3}} ; q^{\frac{2}{3}}\right)_{m}} \\
& =\frac{t q^{\frac{2}{3}}}{f\left(-t^{3} q^{\frac{4}{3}},-t^{-3} q^{\frac{2}{3}}\right)} \sum_{m=-\infty}^{\infty} \frac{(-1)^{m} q^{m^{2}+\frac{7}{3} m} t^{3 m}}{1+q^{2 m+1}} \\
& +\frac{t^{-1} q^{\frac{2}{3}}}{f\left(-t^{-3} q^{\frac{4}{3}},-t^{3} q^{\frac{2}{3}}\right)} \sum_{m=-\infty}^{\infty} \frac{(-1)^{m} q^{m^{2}+\frac{7}{3} m} t^{-3 m}}{1+q^{2 m+1}}+\frac{\left(q^{2} ; q^{2}\right)_{\infty}^{3}}{\left(q^{\frac{2}{3}} ; q^{\frac{2}{3}}\right)_{\infty} f\left(t^{3} q, t^{-3} q\right)} \\
& +\frac{q^{\frac{1}{3}}\left(q^{2} ; q^{2}\right)_{\infty}^{3} f\left(q^{\frac{1}{3}}, q^{\frac{5}{3}}\right)}{\left(q^{\frac{2}{3}} ; q^{\frac{2}{3}}\right)_{\infty} f(q, q) f\left(t^{-3} q, t^{3} q\right)}\left(\frac{t^{-2} f\left(-t^{-3} q^{2},-t^{3}\right)}{f\left(-t^{3} q^{\frac{4}{3}},-t^{-3} q^{\frac{2}{3}}\right)}+\frac{t^{2} f\left(-t^{3} q^{2},-t^{-3}\right)}{f\left(-t^{-3} q^{\frac{4}{3}},-t^{3} q^{\frac{2}{3}}\right)}\right)
\end{aligned}
$$

Proof. Garvan [1988] showed that, for $|q|<|z|<|q|^{-1}$ and $z \neq 1$,

$$
z^{-1}\left(-1+\sum_{m=0}^{\infty} \frac{q^{m^{2}}}{(z ; q)_{m+1}(q / z ; q)_{m}}\right)=\frac{1}{(q ; q)_{\infty}} \sum_{m=-\infty}^{\infty} \frac{(-1)^{m} q^{\frac{3 m(m+1)}{2}}}{1-q^{m} z}
$$

Hickerson [1988, p. 649] remarked that

$$
z^{-1}\left(-1+\sum_{m=0}^{\infty} \frac{q^{m^{2}}}{(z ; q)_{m+1}(q / z ; q)_{m}}\right)=\sum_{m=1}^{\infty} \frac{q^{m(m-1)}}{(z ; q)_{m}\left(z^{-1} q ; q\right)_{m}} .
$$

Combining the two results above, we have

$$
\sum_{m=1}^{\infty} \frac{q^{m(m-1)}}{(z ; q)_{m}\left(z^{-1} q ; q\right)_{m}}=\frac{1}{(q ; q)_{\infty}} \sum_{m=-\infty}^{\infty} \frac{(-1)^{m} q^{\frac{3 m(m+1)}{2}}}{1-z q^{m}} .
$$

We also see this equation in [Gordon and McIntosh 2012, p. 104]. Now, replacing $q$ and $z$ by $q^{2 / 3}$ and $-t q^{1 / 3}$, respectively, (6) becomes

$$
\sum_{m=1}^{\infty} \frac{q^{\frac{2 m(m-1)}{3}}}{\left(-t q^{\frac{1}{3}} ; q^{\frac{2}{3}}\right)_{m}\left(-t^{-1} q^{\frac{1}{3}} ; q^{\frac{2}{3}}\right)_{m}}=\frac{1}{\left(q^{\frac{2}{3}} ; q^{\frac{2}{3}}\right)_{\infty}} \sum_{m=-\infty}^{\infty} \frac{(-1)^{m} q^{m(m+1)}}{1+q^{\frac{2}{3} m+\frac{1}{3}} t} .
$$

In [Choi 2004, p. 378], the author showed that

$$
\sum_{m=-\infty}^{\infty} \frac{(-1)^{m} q^{m(m+1)}}{1-q^{2 m_{z}}}=\frac{\left(q^{2} ; q^{2}\right)_{\infty}^{2}}{\left(z ; q^{2}\right)_{\infty}\left(q^{2} / z ; q^{2}\right)_{\infty}}
$$

which was also recorded by Ramanujan [1988, p. 59] in the lost notebook without proofs. Using (8) with $z$ replaced by $-t^{3} q$, the Jacobi triple product identity and a straightforward calculation show that 
(9)

$$
\begin{aligned}
& \sum_{m=-\infty}^{\infty} \frac{(-1)^{m} q^{m(m+1)}}{1+q^{\frac{2}{3} m+\frac{1}{3} t}}= \sum_{m=-\infty}^{\infty} \frac{(-1)^{m} q^{m(m+1)}\left(1-q^{\frac{2}{3} m+\frac{1}{3}} t+q^{\frac{4}{3} m+\frac{2}{3}} t^{2}\right)}{1+q^{2 m+1} t^{3}} \\
&=\frac{\left(q^{2} ; q^{2}\right)_{\infty}^{3}}{f\left(t^{3} q, t^{-3} q\right)}+\sum_{m=-\infty}^{\infty} \frac{(-1)^{m} q^{m^{2}+\frac{7}{3} m+\frac{2}{3}} t^{-2}}{1+q^{2 m+1} t^{-3}} \\
&+\sum_{m=-\infty}^{\infty} \frac{(-1)^{m} q^{m^{2}+\frac{7}{3} m+\frac{2}{3}} t^{2}}{1+q^{2 m+1} t^{3}}
\end{aligned}
$$

The two sums on the right side of the equation above are Appell-Lerch sums. In his thesis, Zwegers [2002] showed that the normalized Appell-Lerch sum satisfies

$$
\begin{aligned}
\frac{z}{f\left(-h z,-\frac{q}{h z}\right)} \sum_{m=-\infty}^{\infty} \frac{(-1)^{m} q^{\frac{m(m+1)}{2}}(h z)^{m}}{1-q^{m} t z} & -\frac{1}{f\left(-h,-\frac{q}{h}\right)} \sum_{m=-\infty}^{\infty} \frac{(-1)^{m} q^{\frac{m(m+1)}{2}}}{h^{m} 1-q^{m} t} \\
& =-\frac{(q ; q)_{\infty}^{3} f\left(-h t z,-\frac{q}{h t z}\right) f\left(-z,-\frac{q}{z}\right)}{f\left(-t,-\frac{q}{t}\right) f\left(-h,-\frac{q}{h}\right) f\left(-t z,-\frac{q}{t z}\right) f\left(-h z,-\frac{q}{h z}\right)}
\end{aligned}
$$

where $q=e^{2 \pi i \tau}, h=e^{2 \pi i v}, t=e^{2 \pi i u}$ and $z=e^{2 \pi i z^{\prime}}$, such that $\nu, u, z^{\prime} \notin \mathbb{Z}$ and $u, v, u+z^{\prime}, v+z^{\prime} \notin \mathbb{Z} \tau+\mathbb{Z}$. Hence, employing the Jacobi triple product identity, using (7) and (9), applying (10) with $q, t, h$, and $z$ replaced by $q^{2},-q, t^{-3} q^{4 / 3}$, and $t^{3}$, respectively, then again with $q, t, h$, and $z$ replaced by $q^{2},-q, t^{3} q^{4 / 3}$, and $t^{-3}$, respectively, and employing the fact that $f\left(q^{7 / 3}, q^{-1 / 3}\right)=q^{1 / 3} f\left(q^{1 / 3}, q^{5 / 3}\right)$, we obtain Lemma 1 after a slight rearrangement.

Lemma 2. Set $\omega=e^{\frac{2 \pi i}{3}}$. For a complex number $q$ with $|q|<1$, we have

$$
\begin{aligned}
& q^{2} \sum_{m=1}^{\infty} \frac{q^{6 m(m-1)}}{\left(-t q^{3} ; q^{6}\right)_{m}\left(-t^{-1} q^{3} ; q^{6}\right)_{m}} \\
& +\frac{i}{\sqrt{3}}\left\{\frac{1}{f\left(-\omega^{2} t^{-1} q^{2},-\omega^{-2} t\right)} \sum_{m=-\infty}^{\infty} \frac{(-1)^{m} q^{m^{2}+m} \omega^{2 m} t^{-m}}{1+q^{2 m+1}}\right. \\
& \left.+\frac{1}{f\left(-\omega^{2} t q^{2},-\omega^{-2} t^{-1}\right)} \sum_{m=-\infty}^{\infty} \frac{(-1)^{m} q^{m^{2}+m} \omega^{2 m} t^{m}}{1+q^{2 m+1}}-1\right\} \\
& =-\frac{\left(q^{2} ; q^{2}\right)_{\infty}^{3}}{3\left(q^{6} ; q^{6}\right)_{\infty} f\left(t q, t^{-1} q\right)} \\
& +\frac{i}{\sqrt{3}} \frac{\left(q^{2} ; q^{2}\right)_{\infty}^{4} f\left(-t,-t^{-1} q^{2}\right)}{\left(1-\omega^{2}\right) f(q, q) f\left(q, q^{5}\right) f\left(t q, t^{-1} q\right)} \\
& \times\left(\frac{1}{f\left(-\omega^{2} t^{-1} q^{2},-\omega t\right)}-\frac{t^{-1}}{f\left(-\omega^{2} t q^{2},-\omega t^{-1}\right)}\right) .
\end{aligned}
$$


Proof. We first consider the left side of (11). Employing the Jacobi triple product identity, we easily verify that

$$
\begin{gathered}
f\left(-\omega^{2},-\omega q^{2}\right)=\left(1-\omega^{2}\right)\left(q^{6} ; q^{6}\right)_{\infty}, \quad f\left(\omega^{2} q, \omega q\right)=\frac{\left(q^{2} ; q^{2}\right)\left(q^{6} ; q^{6}\right)_{\infty}}{f\left(q, q^{5}\right)}, \\
f\left(-\omega^{2} t^{-1},-\omega t q^{2}\right)=-\omega^{2} t^{-1} f\left(-\omega^{2} t^{-1} q^{2},-\omega t\right) .
\end{gathered}
$$

Then, using (10) with $q, h, t$, and $z$ replaced by $q^{2}, \omega^{2} t^{-1},-q$, and $t$, respectively, applying the equations above, and rearranging terms, we obtain

$$
\begin{aligned}
\frac{1}{f\left(-\omega^{2} t^{-1} q^{2},-\omega t\right)} \sum_{m=-\infty}^{\infty} \frac{(-1)^{m} q^{m(m+1)} \omega^{2 m} t^{-m}}{1+q^{2 m+1}} \\
=\frac{1}{(1-\omega)\left(q^{6} ; q^{6}\right)_{\infty}} \sum_{m=-\infty}^{\infty} \frac{(-1)^{m} q^{m(m+1)} \omega^{2 m}}{1+q^{2 m+1} t} \\
\quad+\frac{\left(q^{2} ; q^{2}\right)_{\infty}^{4} f\left(-t,-t^{-1} q^{2}\right)}{\left(1-\omega^{2}\right) f(q, q) f\left(q, q^{5}\right) f\left(-\omega^{2} t^{-1} q^{2},-\omega t\right) f\left(t q, t^{-1} q\right)} .
\end{aligned}
$$

A straightforward calculation and the Jacobi triple product identity lead us to

$$
\begin{aligned}
\sum_{m=-\infty}^{\infty} & \frac{(-1)^{m} q^{m(m+1)} \omega^{2 m}}{1+q^{2 m+1} t^{-1}} \\
& =\sum_{m=-\infty}^{\infty} \frac{(-1)^{m+1} q^{m(m+1)} \omega^{m+1}\left(q^{2 m+1} t+1-1\right)}{1+q^{2 m+1} t} \\
& =\sum_{m=-\infty}^{\infty}(-1)^{m+1} q^{m(m+1)} \omega^{m+1}+\sum_{m=-\infty}^{\infty} \frac{(-1)^{m} q^{m(m+1)} \omega^{m+1}}{1+q^{2 m+1} t} a \\
& =(1-\omega)\left(q^{6} ; q^{6}\right)_{\infty}+\sum_{m=-\infty}^{\infty} \frac{(-1)^{m} q^{m(m+1)} \omega^{m+1}}{1+q^{2 m+1} t}
\end{aligned}
$$

Using (12), again using (12) with $t$ replaced by $t^{-1}$, and applying (13), we obtain

$$
\begin{aligned}
& \frac{1}{f\left(-\omega^{2} t^{-1} q^{2},-\omega t\right)} \sum_{m=-\infty}^{\infty} \frac{(-1)^{m} q^{m(m+1)} \omega^{2 m} t^{-m}}{1+q^{2 m+1}} \\
& +\frac{1}{f\left(-\omega^{2} t q^{2},-\omega t^{-1}\right)} \sum_{m=-\infty}^{\infty} \frac{(-1)^{m} q^{m(m+1)} \omega^{2 m} t^{m}}{1+q^{2 m+1}}-1 \\
& =\frac{1}{(1-\omega)\left(q^{6} ; q^{6}\right)_{\infty}} \sum_{m=-\infty}^{\infty} \frac{(-1)^{m} q^{m(m+1)}\left(\omega^{2 m}+\omega^{m+1}\right)}{1+q^{2 m+1} t} \\
& +\frac{\left(q^{2} ; q^{2}\right)_{\infty}^{4} f\left(-t,-t^{-1} q^{2}\right)}{\left(1-\omega^{2}\right) f(q, q) f\left(q, q^{5}\right) f\left(t q, t^{-1} q\right)} \\
& \times\left(\frac{1}{f\left(-\omega^{2} t^{-1} q^{2},-\omega t\right)}-\frac{t^{-1}}{f\left(-\omega^{2} t q^{2},-\omega t^{-1}\right)}\right) .
\end{aligned}
$$


We now consider

$$
\begin{aligned}
& \sum_{m=1}^{\infty} \frac{q^{6 m(m-1)+2}}{\left(-t q^{3} ; q^{6}\right)_{m}\left(-t^{-1} q^{3} ; q^{6}\right)_{m}} \\
& \quad+\frac{i}{\sqrt{3}(1-\omega)\left(q^{6} ; q^{6}\right)_{\infty}} \sum_{m=-\infty}^{\infty} \frac{(-1)^{m} q^{m(m+1)}\left(\omega^{2 m}+\omega^{m+1}\right)}{1+q^{2 m+1} t}
\end{aligned}
$$

An elementary calculation shows that $(1+\omega) /(1-\omega)=i / \sqrt{3}$ and $\omega^{2} /(1-\omega)=$ $-i / \sqrt{3}$. Using these, we find that

$$
\begin{aligned}
& \frac{1}{1-\omega} \sum_{m=-\infty}^{\infty} \frac{(-1)^{m} q^{m(m+1)}\left(\omega^{2 m}+\omega^{m+1}\right)}{1+q^{2 m+1} t} \\
&=\frac{i}{\sqrt{3}} \sum_{m=-\infty}^{\infty} \frac{(-1)^{m} q^{9 m^{2}+3 m}}{1+q^{6 m+1} t}+\frac{2 i}{\sqrt{3}} \sum_{m=-\infty}^{\infty} \frac{(-1)^{m} q^{9 m^{2}+9 m+2}}{1+q^{6 m+3} t} \\
&+\frac{i}{\sqrt{3}} \sum_{m=-\infty}^{\infty} \frac{(-1)^{m} q^{9 m^{2}+15 m+6}}{1+q^{6 m+5} t}
\end{aligned}
$$

Therefore, applying (6) with $q$ and $z$ replaced by $q^{6}$ and $-t q^{3}$, respectively, (16), and (8) with $z$ replaced by $-t q$, we deduce that (15) equals

$$
\begin{aligned}
& \frac{1}{\left(q^{6} ; q^{6}\right)_{\infty}} \sum_{m=-\infty}^{\infty} \frac{(-1)^{m} q^{9 m^{2}+9 m+2}}{1+q^{6 m+3} t}-\frac{1}{3\left(q^{6} ; q^{6}\right)_{\infty}} \sum_{m=-\infty}^{\infty} \frac{(-1)^{m} q^{9 m^{2}+3 m}}{1+q^{6 m+1} t} \\
& -\frac{2}{3\left(q^{6} ; q^{6}\right)_{\infty}} \sum_{m=-\infty}^{\infty} \frac{(-1)^{m} q^{9 m^{2}+9 m+2}}{1+q^{6 m+3} t} \\
& \quad-\frac{1}{3\left(q^{6} ; q^{6}\right)_{\infty}} \sum_{m=-\infty}^{\infty} \frac{(-1)^{m} q^{9 m^{2}+15 m+6}}{1+q^{6 m+5} t} \\
& =-\frac{1}{3\left(q^{6} ; q^{6}\right)_{\infty}} \sum_{m=-\infty}^{\infty} \frac{(-1)^{m} q^{m^{2}+m}}{1+q^{2 m+1} t} \\
& =-\frac{1}{3\left(q^{6} ; q^{6}\right)_{\infty}} \frac{\left(q^{2} ; q^{2}\right)_{\infty}^{3}}{f\left(t q, t^{-1} q\right)} .
\end{aligned}
$$

In conclusion, by combining (14) and (17) we have derived Lemma 2.

\section{The proofs of the first identity}

First proof of Theorem 1. By a simple calculation and integration by substitution, we obtain 


$$
\begin{aligned}
& \int_{0}^{\infty} \frac{e^{-\frac{\pi n x^{2}}{3}} \cos \pi t x}{e^{\frac{2 \pi x}{3}}+1+e^{-\frac{2 \pi x}{3}} d x} \\
&=\frac{\sqrt{3}}{4 i} e^{-\frac{3 \pi t^{2}}{4 n}}\left\{e^{\frac{\pi i}{3}} \int_{-\infty-i \frac{t}{2 n}}^{\infty-i \frac{t}{2 n}} \frac{e^{-3 \pi n y^{2}}}{e^{2 \pi\left(y+i \frac{t}{2 n}\right)}+e^{\frac{\pi i}{3}}} d y\right. \\
&+e^{\frac{\pi i}{3}} \int_{-\infty+i \frac{t}{2 n}}^{\infty+i \frac{t}{2 n}} \frac{e^{-3 \pi n y^{2}}}{e^{2 \pi\left(y-i \frac{t}{2 n}\right)}+e^{\frac{\pi i}{3}}} d y \\
&-e^{-\frac{\pi i}{3}} \int_{-\infty-i \frac{t}{2 n}}^{\infty-i \frac{t}{2 n}} \frac{e^{-3 \pi n y^{2}}}{e^{2 \pi\left(y+i \frac{t}{2 n}\right)}+e^{-\frac{\pi i}{3}}} d y \\
&\left.-e^{-\frac{\pi i}{3}} \int_{-\infty+i \frac{t}{2 n}}^{\infty+i \frac{t}{2 n}} \frac{e^{-3 \pi n y^{2}}}{e^{2 \pi\left(y-i \frac{t}{2 n}\right)}+e^{-\frac{\pi i}{3}}} d y\right\}
\end{aligned}
$$

For a sufficiently large positive number $d$, we consider the integral

$$
\int_{\gamma} \frac{e^{-3 \pi n y^{2}}}{e^{2 \pi\left(y+i \frac{t}{2 n}\right)}+e^{\frac{\pi i}{3}}} d y
$$

taken around the rectangle $\gamma$ whose vertices are at the points $\pm d$ and $\pm d-i \frac{t}{2 n}$. We easily verify that

$$
\frac{e^{-3 \pi n y^{2}}}{e^{2 \pi\left(y+i \frac{t}{2 n}\right)}+e^{\frac{\pi i}{3}}}
$$

has simple poles at $i\left(-\frac{t}{2 n}+\frac{2}{3}+k\right)$ for integers $k$. Assume that $\Re\left(\frac{t}{n}\right)>0$. Since $\Re\left(\frac{t}{2 n}\right) \pm \frac{1}{3} \notin \mathbb{Z}$ and $\Re\left(\frac{t}{2 n}\right) \pm \frac{2}{3} \notin \mathbb{Z}$, after some elementary manipulation and employing Cauchy's residue theorem, we find that

$$
\begin{gathered}
\int_{-d-i \frac{t}{2 n}}^{d-i \frac{t}{2 n}} \frac{e^{-3 \pi n y^{2}}}{e^{2 \pi\left(y+i \frac{t}{2 n}\right)}+e^{\frac{\pi i}{3}}} d y \\
=\sum_{0 \leq k<\Re \frac{t}{2 n}-\frac{2}{3}} i e^{\frac{2 \pi i}{3}+\frac{3 \pi t^{2}}{4 n}-2 \pi t-3 \pi t k} q^{-\frac{4}{9}-k^{2}-\frac{4}{3} k} \\
\quad+\left(\int_{-d}^{d}+\int_{d}^{d-i \frac{t}{2 n}}+\int_{-d-i \frac{t}{2 n}}^{-d}\right) \frac{e^{-3 \pi n y^{2}}}{e^{2 \pi\left(y+i \frac{t}{2 n}\right)}+e^{\frac{\pi i}{3}}} d y .
\end{gathered}
$$

Since

$$
\left|\left(\int_{d}^{d-i \frac{t}{2 n}}+\int_{-d-i \frac{t}{2 n}}^{-d}\right) \frac{e^{-3 \pi n y^{2}}}{e^{2 \pi\left(y+i \frac{t}{2 n}\right)}+e^{\frac{\pi i}{3}}} d y\right| \leq 2 \frac{e^{-3 \pi n\left(d^{2}-\left(\frac{t}{2 n}\right)^{2}\right)}}{e^{2 \pi d}-1} \frac{|t|}{n},
$$

we find that the sum of these integrals tends to 0 as $d$ tends to $\infty$. Thus, letting $d \rightarrow \infty$ we verify that 


$$
\begin{aligned}
& \int_{-\infty-i \frac{t}{2 n}}^{\infty-i \frac{t}{2 n}} \frac{e^{-3 \pi n y^{2}}}{e^{2 \pi\left(y+i \frac{t}{2 n}\right)}+e^{\frac{\pi i}{3}}} d y \\
& \quad=\sum_{0 \leq k<\Re \frac{t}{2 n}-\frac{2}{3}} i e^{\frac{2 \pi i}{3}+\frac{3 \pi t^{2}}{4 n}-2 \pi t-3 \pi t k} q^{-\frac{4}{9}-k^{2}-\frac{4}{3} k}+\int_{-\infty}^{\infty} \frac{e^{-3 \pi n y^{2}}}{e^{2 \pi\left(y+i \frac{t}{2 n}\right)}+e^{\frac{\pi i}{3}}} d y .
\end{aligned}
$$

We can also establish similar results for the other three integrals in (18) for $\Re\left(\frac{t}{n}\right)>0$. We then apply these results to (18) and collect the sums to obtain

$$
\begin{aligned}
& -\sum_{0 \leq k<\Re \frac{t}{2 n}-\frac{2}{3}} e^{-2 \pi t-3 \pi t k} q^{-\frac{4}{9}-k^{2}-\frac{4}{3} k}-\sum_{-\Re \frac{t}{2 n}-\frac{2}{3}<k \leq-1} e^{2 \pi t+3 \pi t k} q^{-\frac{4}{9}-k^{2}-\frac{4}{3} k} \\
& \quad+\sum_{0 \leq k<\Re \frac{t}{2 n}-\frac{1}{3}} e^{-\pi t-3 \pi t k} q^{-\frac{1}{9}-k^{2}-\frac{2}{3} k}+\sum_{-\Re \frac{t}{2 n}-\frac{1}{3}<k \leq-1} e^{\pi t+3 \pi t k} q^{-\frac{1}{9}-k^{2}-\frac{2}{3} k} .
\end{aligned}
$$

Replacing $k$ by $-k-1$ in the second and fourth sums above, we find that the four sums above cancel. Thus, for $\Re\left(\frac{t}{n}\right)>0$,

$$
\begin{aligned}
\int_{0}^{\infty} & \frac{e^{-\frac{\pi n x^{2}}{3}} \cos \pi t x}{e^{\frac{2 \pi x}{3}}+1+e^{-\frac{2 \pi x}{3}}} d x \\
= & \frac{\sqrt{3}}{4 i} e^{-\frac{3 \pi t^{2}}{4 n}}\left\{e^{\frac{\pi i}{3}} \int_{-\infty}^{\infty}\left(\frac{e^{-3 \pi n y^{2}}}{e^{2 \pi\left(y+i \frac{t}{2 n}\right)}+e^{\frac{\pi i}{3}}}+\frac{e^{-3 \pi n y^{2}}}{e^{2 \pi\left(y-i \frac{t}{2 n}\right)}+e^{\frac{\pi i}{3}}}\right) d y\right. \\
& \left.\quad-e^{-\frac{\pi i}{3}} \int_{-\infty}^{\infty}\left(\frac{e^{-3 \pi n y^{2}}}{e^{2 \pi\left(y+i \frac{t}{2 n}\right)}+e^{-\frac{\pi i}{3}}}+\frac{e^{-3 \pi n y^{2}}}{e^{2 \pi\left(y-i \frac{t}{2 n}\right)}+e^{-\frac{\pi i}{3}}}\right) d y\right\} .
\end{aligned}
$$

For $\Re\left(\frac{t}{n}\right)<0$, a similar process also brings us to (19). Also, for $\Re\left(\frac{t}{n}\right)=0$, we directly derive (19) from (18). Therefore, for any positive number $n$ and any number $t$ such that $\Re\left(\frac{t}{n}\right) \pm \frac{1}{3} \notin \mathbb{Z}$ and $\Re\left(\frac{t}{n}\right) \pm \frac{2}{3} \notin \mathbb{Z}$, we obtain (19).

Next we must evaluate the integrals on the right side of (19). We need the modular transformation formula for $\theta_{11}$

$$
\theta_{11}\left(\frac{x}{\tau},-\frac{1}{\tau}\right)=-i \sqrt{-i \tau} e^{\pi i x^{2} / \tau} \theta_{11}(x, \tau) .
$$

Additionally, $F(x, \tau)$ and $\theta_{11}$ satisfy the transformation formulas

$$
\begin{aligned}
\theta_{11}(x, \tau) & =-\theta_{11}(x+1, \tau)=-\theta_{11}(-x, \tau)=-e^{\pi i(2 x+\tau)} \theta_{11}(x+\tau, \tau), \\
F(x, \tau) & =-F(x+1, \tau)=-F(x+\tau, \tau)+\theta_{11}(x, \tau)=-F(-x+\tau, \tau) \\
& =F(-x, \tau)+\theta_{11}(x, \tau) .
\end{aligned}
$$

We employ these formulas to evaluate the four integrals on the right-hand side of (19). Recall that $q_{1}=e^{-\frac{\pi}{3 n}}$ and $q=e^{-3 \pi n}$. We first consider the first integral on 
the right side of (19). Replacing $\tau, x$, and $\theta$ by $3 i n, 0$, and $\frac{2}{3}-\frac{t}{2 n}$, respectively, in Mordell's formula (1), we find that

$$
\begin{gathered}
\int_{-\infty}^{\infty} \frac{e^{-3 \pi n y^{2}}}{e^{2 \pi\left(y+i \frac{t}{2 n}\right)}-e^{\frac{4 \pi i}{3}}} d y=e^{-\frac{\pi i t}{n}} \int_{-\infty}^{\infty} \frac{e^{-3 \pi n y^{2}}}{e^{2 \pi y}-e^{2 \pi i\left(\frac{2}{3}-\frac{t}{2 n}\right)} d y} \\
=e^{\frac{3 \pi t^{2}}{4 n}-2 \pi t-\frac{4 \pi i}{3}} q^{-\frac{4}{9}} \frac{F\left(\frac{2}{3}-\frac{t}{2 n},-\frac{1}{3 i n}\right)-3 n F\left(2 i n-i \frac{3 t}{2}, 3 i n\right)}{3 i n \theta_{11}\left(2 i n-i \frac{3 t}{2}, 3 i n\right)} .
\end{gathered}
$$

We are able to establish a similar result for each of the remaining three integrals. From (20), we deduce that

$$
\begin{aligned}
\theta_{11}\left(2 i n-\frac{3}{2} i t, 3 i n\right) & =\frac{i}{\sqrt{3 n}} e^{\frac{4}{3} \pi n-2 \pi t+\frac{3 \pi t^{2}}{4 n}} \theta_{11}\left(\frac{2}{3}-\frac{t}{2 n},-\frac{1}{3 i n}\right), \\
\theta_{11}\left(i n-\frac{3}{2} i t, 3 i n\right) & =\frac{i}{\sqrt{3 n}} e^{\frac{1}{3} \pi n-\pi t+\frac{3 \pi t^{2}}{4 n}} \theta_{11}\left(\frac{1}{3}-\frac{t}{2 n},-\frac{1}{3 i n}\right) .
\end{aligned}
$$

Using the evaluations of the four integrals, employing the above modular transformations for $\theta_{11}$ and the formulas satisfied by $\theta_{11}$ and $F$, simplifying terms, and employing the definitions of $\theta_{11}$ and $F$, we obtain

$$
\begin{aligned}
\frac{2}{\sqrt{3}} \int_{0}^{\infty} \frac{e^{-\frac{\pi n x^{2}}{3}} \cos \pi t x}{e^{\frac{2 \pi x}{3}}+1+e^{-\frac{2 \pi x}{3}}} d x \\
=\frac{e^{\pi t} q^{\frac{8}{9}}}{f\left(-e^{3 \pi t} q^{\frac{4}{3}},-e^{-3 \pi t} q^{\frac{2}{3}}\right)} \sum_{m=-\infty}^{\infty} \frac{(-1)^{m} q^{m^{2}+\frac{7}{3} m} e^{3 m \pi t}}{1+q^{2 m+1}} \\
+\frac{e^{-\pi t} q^{\frac{8}{9}}}{f\left(-e^{-3 \pi t} q^{\frac{4}{3}},-e^{3 \pi t} q^{\frac{2}{3}}\right)} \sum_{m=-\infty}^{\infty} \frac{(-1)^{m} q^{m^{2}+\frac{7}{3} m} e^{-3 m \pi t}}{1+q^{2 m+1}} \\
\quad \times\left\{\frac{1}{f\left(-\omega^{2} e^{-\frac{\pi i t}{n}} q_{1}^{2},-\omega^{-2} e^{\frac{\pi i t}{n}}\right)} \sum_{m=-\infty}^{\infty} \frac{(-1)^{m} q_{1}^{m^{2}+m} \omega^{2 m} e^{-\frac{m \pi i t}{n}}}{1+q_{1}^{2 m+1}}\right. \\
\left.\quad+\frac{1}{f\left(-\omega^{2} e^{\frac{\pi i t}{n}} q_{1}^{2},-\omega^{-2} e^{-\frac{\pi i t}{n}}\right)} \sum_{m=-\infty}^{\infty} \frac{(-1)^{m} q_{1}^{m^{2}+m} \omega^{2 m} e^{\frac{m \pi i t}{n}}}{1+q_{1}^{2 m+1}}-1\right\} .
\end{aligned}
$$

We are now ready to complete the proof. Use Lemma 1 with $t$ replaced by $e^{\pi t}$ and employ Lemma 2 with $q$ and $t$ replaced by $q_{1}$ and $e^{\frac{\pi i t}{n}}$, respectively. After some elementary manipulations, we find that the sum of the new left-hand sides of Lemma 1 and (11) equals 
(22)

$$
\begin{aligned}
& q^{\frac{2}{9}} \sum_{m=1}^{\infty} \frac{q^{\frac{2 m(m-1)}{3}}}{\left(-e^{\pi t} q^{\frac{1}{3}} ; q^{\frac{2}{3}}\right)_{m}\left(-e^{-\pi t} q^{\frac{1}{3}} ; q^{\frac{2}{3}}\right)_{m}} \\
& +\frac{e^{-\frac{3 \pi t^{2}}{4 n}} q_{1}^{2}}{\sqrt{n}} \sum_{m=1}^{\infty} \frac{q_{1}^{6 m(m-1)}}{\left(-e^{i \frac{\pi t}{n}} q_{1}^{3} ; q_{1}^{6}\right)_{m}\left(-e^{-i \frac{\pi t}{n}} q_{1}^{3} ; q_{1}^{6}\right)_{m}} \\
& -\frac{e^{\pi t} q^{\frac{8}{9}}}{f\left(-e^{3 \pi t} q^{\frac{4}{3}},-e^{-3 \pi t} q^{\frac{2}{3}}\right)} \sum_{m=-\infty}^{\infty} \frac{(-1)^{m} q^{m^{2}+\frac{7}{3} m} e^{3 m \pi t}}{1+q^{2 m+1}} \\
& -\frac{e^{-\pi t} q^{\frac{8}{9}}}{f\left(-e^{-3 \pi t} q^{\frac{4}{3}},-e^{3 \pi t} q^{\frac{2}{3}}\right)} \sum_{m=-\infty}^{\infty} \frac{(-1)^{m} q^{m^{2}+\frac{7}{3} m} e^{-3 m \pi t}}{1+q^{2 m+1}} \\
& +i \frac{e^{-\frac{3 \pi t^{2}}{4 n}}}{\sqrt{3 n}}\left\{\frac{\sum^{\infty} \frac{(-1)^{m} q_{1}^{m^{2}+m} \omega^{2 m} e^{-\frac{m \pi i t}{n}}}{f\left(-\omega^{2} e^{-\frac{\pi i t}{n}} q_{1}^{2},-\omega^{-2} e^{\frac{\pi i t}{n}}\right)}}{m_{m}}\right. \\
& \left.+\frac{1}{f\left(-\omega^{2} e^{\frac{\pi i t}{n}} q_{1}^{2},-\omega^{-2} e^{-\frac{\pi i t}{n}}\right)} \sum_{m=-\infty}^{\infty} \frac{(-1)^{m} q_{1}^{m^{2}+m} \omega^{2 m} e^{\frac{m \pi i t}{n}}}{1+q_{1}^{2 m+1}}-1\right\}
\end{aligned}
$$

and the sum of the new right-hand sides of Lemma 1 and (11) equals (23)

$$
\begin{aligned}
& q^{\frac{2}{9}}\left\{\frac{\left(q^{2} ; q^{2}\right)_{\infty}^{3}}{\left(q^{\frac{2}{3}} ; q^{\frac{2}{3}}\right)_{\infty} f\left(e^{3 \pi t} q, e^{-3 \pi t} q\right)}+\frac{q^{\frac{1}{3}}\left(q^{2} ; q^{2}\right)_{\infty}^{3} f\left(q^{\frac{1}{3}}, q^{\frac{5}{3}}\right) f\left(-e^{-3 \pi t} q^{2},-e^{3 \pi t}\right)}{\left(q^{\frac{2}{3}} ; q^{\frac{2}{3}}\right)_{\infty} f(q, q) f\left(e^{-3 \pi t} q, e^{3 \pi t} q\right)}\right. \\
& \left.\times\left(\frac{e^{-2 \pi t}}{f\left(-e^{3 \pi t} q^{\frac{4}{3}},-e^{-3 \pi t} q^{\frac{2}{3}}\right)}-\frac{e^{-\pi t}}{f\left(-e^{-3 \pi t} q^{\frac{4}{3}},-e^{3 \pi t} q^{\frac{2}{3}}\right)}\right)\right\} \\
& +\frac{e^{-\frac{3 \pi t^{2}}{4 n}}}{\sqrt{n}}\left\{-\frac{\left(q_{1}^{2} ; q_{1}^{2}\right)_{\infty}^{3}}{3\left(q_{1}^{6} ; q_{1}^{6}\right)_{\infty} f\left(e^{\frac{\pi i t}{n}} q_{1}, e^{-\frac{\pi i t}{n}} q_{1}\right)}\right. \\
& +\frac{i}{\sqrt{3}} \frac{\left(q_{1}^{2} ; q_{1}^{2}\right)_{\infty}^{4} f\left(-e^{\frac{\pi i t}{n}},-e^{-\frac{\pi i t}{n}} q_{1}^{2}\right)}{\left(1-\omega^{2}\right) f\left(q_{1}, q_{1}\right) f\left(q_{1}, q_{1}^{5}\right) f\left(e^{\frac{\pi i t}{n}} q_{1}, e^{-\frac{\pi i t}{n}} q_{1}\right)} \\
& \left.\times\left(\frac{1}{f\left(-\omega^{2} e^{-\frac{\pi i t}{n}} q_{1}^{2},-\omega e^{\frac{\pi i t}{n}}\right)}-\frac{e^{-\frac{\pi i t}{n}}}{f\left(-\omega^{2} e^{\frac{\pi i t}{n}} q_{1}^{2},-\omega e^{-\frac{\pi i t}{n}}\right)}\right)\right\} .
\end{aligned}
$$

Next we prove that (23) is identically equal to zero. Using the definition of $\theta_{11}$, the Jacobi triple product identity, and the transformation formula (20) for $\theta_{11}$, we deduce the following formula for Ramanujan's theta function $f$ :

$$
f\left(-e^{2 \pi i(x+\tau)},-e^{-2 \pi i x}\right)=\frac{i}{\sqrt{-i \tau}} e^{-\pi i\left(x+\frac{\tau}{4}+\frac{x^{2}-x}{\tau}+\frac{1}{4 \tau}\right)} f\left(-e^{2 \pi i \frac{x-1}{\tau}},-e^{-2 \pi i \frac{x}{\tau}}\right) .
$$


Set $\tau=3 i n$, and recall that $q_{1}=e^{-\frac{\pi}{3 n}}$ and $q=e^{-3 \pi n}$ to obtain (24)

$$
f\left(-e^{2 \pi i x} q^{2},-e^{-2 \pi i x}\right)=\frac{i}{\sqrt{3 n}} q^{-\frac{1}{4}} e^{-\pi i x-\frac{\pi}{3 n}\left(x-\frac{1}{2}\right)^{2}} f\left(-e^{\frac{2 \pi x}{3 n}} q_{1}^{2},-e^{-\frac{2 \pi x}{3 n}}\right) .
$$

Since $\lim _{x \rightarrow 0}\left(1-e^{-\frac{2 \pi}{3 n} x}\right) /\left(1-e^{-2 \pi i x}\right)=-i /(3 n)$, dividing both sides of (24) by $1-e^{-2 \pi i x}$ and tending $x$ to 0 leads us to find that

$$
\left(q^{2} ; q^{2}\right)_{\infty}^{3}=\frac{1}{3 n \sqrt{3 n}} q^{-\frac{1}{4}} q_{1}^{\frac{1}{4}}\left(q_{1}^{2} ; q_{1}^{2}\right)_{\infty}^{3}
$$

Applying (24) twice with $x=\frac{1}{2}-\frac{3}{2} i n-\frac{3}{2} i t$ and $x=-i n$, respectively, (25), and the fact that $i \sqrt{3}(1-\omega) e^{-\frac{\pi i}{3}}=3$, and employing the Jacobi triple product identity, we obtain

$$
\frac{q^{\frac{2}{9}}\left(q^{2} ; q^{2}\right)_{\infty}^{3}}{\left(q^{\frac{2}{3}} ; q^{\frac{2}{3}}\right)_{\infty} f\left(e^{3 \pi t} q, e^{-3 \pi t} q\right)}=\frac{e^{-\frac{3 \pi t^{2}}{4 n}}}{\sqrt{n}} \frac{\left(q_{1}^{2} ; q_{1}^{2}\right)_{\infty}^{3}}{3\left(q_{1}^{6} ; q_{1}^{6}\right)_{\infty} f\left(e^{\frac{\pi i t}{n}} q_{1}, e^{-\frac{\pi i t}{n}} q_{1}\right)}
$$

Applying (24) with $x$ replaced by $\frac{1}{2}-\frac{1}{2} i n, \frac{3 i t}{2}, \frac{1}{2}-\frac{3}{2} i n,-\frac{3}{2} i t-i n$, and $\frac{3}{2} i t-i n$, respectively, and employing the Jacobi triple product,we obtain

$$
f\left(q^{\frac{5}{3}}, q^{\frac{1}{3}}\right)=\frac{1}{\sqrt{3 n}} q^{-\frac{1}{9}} f\left(-e^{-\frac{\pi i}{3}} q_{1},-e^{\frac{\pi i}{3}} q_{1}\right)=\frac{1}{\sqrt{3 n}} q^{-\frac{1}{9}} \frac{\left(q_{1}^{2} ; q_{1}^{2}\right)_{\infty}\left(q_{1}^{6} ; q_{1}^{6}\right)_{\infty}}{f\left(q_{1}, q_{1}^{5}\right)},
$$

$$
\begin{gathered}
f\left(-e^{-3 \pi t} q^{2},-e^{3 \pi t}\right)=-\frac{i q^{-\frac{1}{4}} q_{1}^{\frac{1}{4}} e^{\frac{3 \pi t}{2}+\frac{3 \pi t^{2}}{4 n}-\frac{\pi i t}{2 n}}}{\sqrt{3 n}} f\left(-e^{\frac{\pi i t}{n}},-e^{-\frac{\pi i t}{n}} q_{1}^{2}\right), \\
f(q, q)=\frac{1}{\sqrt{3 n}} f\left(q_{1}, q_{1}\right),
\end{gathered}
$$

$$
\begin{array}{r}
f\left(-e^{3 \pi t} q^{\frac{4}{3}},-e^{-3 \pi t} q^{\frac{2}{3}}\right) \\
=\frac{i q^{-\frac{1}{36}} q_{1}^{\frac{1}{4}} e^{-\frac{\pi i}{3}-\frac{\pi t}{2}+\frac{3 \pi t^{2}}{4 n}-\frac{\pi i t}{2 n}}}{\sqrt{3 n}} f\left(-e^{-\frac{\pi i t}{n}} \omega^{2} q_{1}^{2},-e^{\frac{\pi i t}{n}} \omega\right), \\
f\left(-e^{-3 \pi t} q^{\frac{4}{3}},-e^{3 \pi t} q^{\frac{2}{3}}\right) \\
=\frac{i q^{-\frac{1}{36}} q_{1}^{\frac{1}{4}} e^{-\frac{\pi i}{3}+\frac{\pi t}{2}+\frac{3 \pi t^{2}}{4 n}+\frac{\pi i t}{2 n}}}{\sqrt{3 n}} f\left(-e^{\frac{\pi i t}{n}} \omega^{2} q_{1}^{2},-e^{-\frac{\pi i t}{n}} \omega\right) .
\end{array}
$$

Employing (26)-(31) and using the fact that $e^{\frac{\pi i}{3}}=\sqrt{3} i /\left(1-\omega^{2}\right)$, we conclude that 


$$
\begin{aligned}
& \frac{q^{\frac{5}{9}}\left(q^{2} ; q^{2}\right)_{\infty}^{3} f\left(q^{\frac{1}{3}}, q^{\frac{5}{3}}\right) f\left(-e^{-3 \pi t} q^{2},-e^{3 \pi t}\right)}{\left(q^{\frac{2}{3}} ; q^{\frac{2}{3}}\right)_{\infty} f(q, q) f\left(e^{-3 \pi t} q, e^{3 \pi t} q\right)} \\
& \quad \times\left(\frac{e^{-2 \pi t}}{f\left(-e^{3 \pi t} q^{\frac{4}{3}},-e^{-3 \pi t} q^{\frac{2}{3}}\right)}-\frac{e^{-\pi t}}{f\left(-e^{-3 \pi t} q^{\frac{4}{3}},-e^{3 \pi t} q^{\frac{2}{3}}\right)}\right) \\
& =-\frac{e^{-\frac{3 \pi t^{2}}{4 n}}}{\sqrt{n}} \frac{i}{\sqrt{3}} \frac{\left(q_{1}^{2} ; q_{1}^{2}\right)_{\infty}^{4} f\left(-e^{\frac{\pi i t}{n}},-e^{-\frac{\pi i t}{n}} q_{1}^{2}\right)}{\left(1-\omega^{2}\right) f\left(q_{1}, q_{1}\right) f\left(q_{1}, q_{1}^{5}\right) f\left(e^{\frac{\pi i t}{n}} q_{1}, e^{-\frac{\pi i t}{n}} q_{1}\right)} \\
& \times\left(\frac{1}{f\left(-\omega^{2} e^{-\frac{\pi i t}{n}} q_{1}^{2},-\omega e^{\frac{\pi i t}{n}}\right)}-\frac{e^{-\frac{\pi i t}{n}}}{f\left(-\omega^{2} e^{\frac{\pi i t}{n}} q_{1}^{2},-\omega e^{-\frac{\pi i t}{n}}\right)}\right) .
\end{aligned}
$$

As a result, combining (26) and (32), we know that (23) equals 0 . Thus, (22) equals 0 . Therefore, comparing (21) and (22), we have proved Theorem 1.

Second proof of Theorem 1. We now consider the equation

$$
\frac{1}{\sqrt{3}} \int_{-\infty}^{\infty} \frac{e^{\frac{\pi i \tau x^{2}}{3}-2 \pi z x}}{e^{\frac{2 \pi x}{3}}+1+e^{-\frac{2 \pi x}{3}}} d x=g(z ; \tau)+\frac{e^{\frac{3 \pi i z^{2}}{\tau}}}{\sqrt{-i \tau}} g\left(-\frac{z}{\tau} ;-\frac{1}{\tau}\right)
$$

where

$$
g(z ; \tau):=\frac{e^{\frac{2 \pi i \tau}{3}}}{\left(e^{2 \pi i \tau} ; e^{2 \pi i \tau}\right)_{\infty}} \sum_{m=-\infty}^{\infty} \frac{(-1)^{m} e^{3 \pi i \tau m(m+1)}}{1+e^{2 \pi i z+2 \pi i \tau\left(m+\frac{1}{2}\right)}} .
$$

Comparing the definitions of $g$ and $g_{3}$, we find that

$$
g(z ; \tau)=e^{\frac{2 \pi i \tau}{3}} g_{3}\left(e^{2 \pi i z+\pi i(\tau+1)}, e^{2 \pi i \tau}\right) .
$$

We now set $\tau=i n, q=e^{-2 \pi n}$, and $q_{1}=e^{-\frac{2 \pi}{n}}$. Using (6) with $z$ replaced by $e^{2 \pi i z}$, we get

$$
\begin{aligned}
g(z ; i n) & =\frac{q^{\frac{1}{3}}}{(q ; q)_{\infty}} \sum_{m=-\infty}^{\infty} \frac{(-1)^{m} q^{\frac{3 m(m+1)}{2}}}{1+e^{2 \pi i z} q^{\left(m+\frac{1}{2}\right)}} \\
& =q^{\frac{1}{3}} \sum_{m=1}^{\infty} \frac{q^{m(m-1)}}{\left(-e^{2 \pi i z} q^{\frac{1}{2}} ; q\right)_{m}\left(-e^{-2 \pi i z} q^{\frac{1}{2}} ; q\right)_{m}}
\end{aligned}
$$

Similarly, we obtain

$$
g\left(\frac{i z}{n} ; \frac{i}{n}\right)=q_{1}^{\frac{1}{3}} \sum_{m=1}^{\infty} \frac{q_{1}^{m(m-1)}}{\left(-e^{-\frac{2 \pi z}{n}} q_{1}^{\frac{1}{2}} ; q_{1}\right)_{m}\left(-e^{\frac{2 \pi z}{n}} q_{1}^{\frac{1}{2}} ; q_{1}\right)_{m}} .
$$

Applying these results to (33), we easily verify that proving (33) is equivalent to proving the equation in Theorem 1. So, we prove (33) instead of Theorem 1. 
We first discuss the right-hand side of (33). From the definition of $g(z ; \tau)$, we see that $g(z ; \tau)$ is a meromorphic function of $z$ with simple poles in $\left(\frac{1}{2}+\mathbb{Z}\right) \tau+$ $\frac{1}{2}+\mathbb{Z}$. By a direct calculation, we can determine that its residue at $-\frac{1}{2} \tau-\frac{1}{2}$ is $-q^{1 / 3} /\left(2 \pi i(q ; q)_{\infty}\right)$. We will find two functional equations for the function $g(z ; \tau)$. By the definition of $g(z ; \tau)$, we easily get

$$
g(z+1 ; \tau)=g(z ; \tau) .
$$

Using the definition of $g(z ; \tau)$ and the Jacobi triple product identity, we obtain

$$
\begin{aligned}
g(z+\tau ; \tau) & =\frac{e^{\frac{5 \pi i \tau}{3}+2 \pi i z}}{\left(e^{2 \pi i \tau} ; e^{2 \pi i \tau}\right)_{\infty}} \sum_{m=-\infty}^{\infty} \frac{(-1)^{m} e^{\pi i \tau\left(3 m^{2}-m\right)}}{1+e^{2 \pi i z+2 \pi i \tau\left(m+\frac{1}{2}\right)}} \\
& =e^{\frac{5 \pi i \tau}{3}+2 \pi i z}-\frac{e^{\frac{8 \pi i \tau}{3}}+4 \pi i z}{\left(e^{2 \pi i \tau} ; e^{2 \pi i \tau}\right)_{\infty}} \sum_{m=-\infty}^{\infty} \frac{(-1)^{m} e^{\pi i \tau\left(3 m^{2}+m\right)}}{1+e^{2 \pi i z+2 \pi i \tau\left(m+\frac{1}{2}\right)}} \\
& =e^{\frac{5 \pi i \tau}{3}+2 \pi i z}-e^{\frac{8 \pi i \tau}{3}+4 \pi i z}+e^{3 \pi i \tau+6 \pi i z} g(z ; \tau) .
\end{aligned}
$$

In particular,

$$
g(z+\tau ; \tau)-e^{3 \pi i \tau+6 \pi i z} g(z ; \tau)=e^{\frac{5 \pi i \tau}{3}+2 \pi i z}-e^{\frac{8 \pi i \tau}{3}+4 \pi i z} .
$$

Let $G(z ; \tau)$ denote the right-hand side of (33). Then, using the functional equations (34) and (35), we get

$$
\begin{aligned}
G(z+1 ; \tau)= & g(z+1 ; \tau)+\frac{e^{\frac{3 \pi i(z+1)^{2}}{\tau}}}{\sqrt{-i \tau}} g\left(-\frac{z+1}{\tau} ;-\frac{1}{\tau}\right) \\
= & g(z ; \tau)+\frac{e^{\frac{3 \pi i(z+1)^{2}}{\tau}}}{\sqrt{-i \tau}}\left\{e^{-\frac{5 \pi i}{3 \tau}-\frac{2 \pi i z}{\tau}}-e^{-\frac{8 \pi i}{3 \tau}-\frac{4 \pi i z}{\tau}}\right. \\
& \left.+e^{-\frac{3 \pi i}{\tau}-\frac{6 \pi i z}{\tau}} g\left(-\frac{z}{\tau} ;-\frac{1}{\tau}\right)\right\} .
\end{aligned}
$$

Thus,

$$
G(z+1 ; \tau)-G(z ; \tau)=\frac{e^{\frac{3 \pi i(z+1)^{2}}{\tau}}}{\sqrt{-i \tau}}\left(e^{-\frac{5 \pi i}{3 \tau}-\frac{2 \pi i z}{\tau}}-e^{-\frac{8 \pi i}{3 \tau}-\frac{4 \pi i z}{\tau}}\right) .
$$

Again, using the functional equations (34) and (35), we obtain

$$
\begin{aligned}
G(z+\tau ; \tau)= & g(z+\tau ; \tau)+\frac{e^{\frac{3 \pi i(z+\tau)^{2}}{\tau}}}{\sqrt{-i \tau}} g\left(-\frac{z+\tau}{\tau} ;-\frac{1}{\tau}\right) \\
=e^{\frac{5 \pi i \tau}{3}+2 \pi i z}-e^{\frac{8 \pi i \tau}{3}+4 \pi i z}+e^{3 \pi i \tau+6 \pi i z} g(z ; \tau) & \\
& +\frac{e^{\frac{3 \pi i z^{2}}{\tau}+6 \pi i z+3 \pi i \tau}}{\sqrt{-i \tau}} g\left(-\frac{z}{\tau} ;-\frac{1}{\tau}\right) .
\end{aligned}
$$


So,

$$
G(z+\tau ; \tau)-e^{3 \pi i \tau+6 \pi i z} G(z ; \tau)=e^{\frac{5 \pi i \tau}{3}+2 \pi i z}-e^{\frac{8 \pi i \tau}{3}+4 \pi i z} .
$$

Therefore, $G(z ; \tau)$ satisfies the functional equations (36) and (37). Recall that the residue of the function $g(z ; \tau)$ at $-\frac{1}{2} \tau-\frac{1}{2}$ is $-q^{1 / 3} /\left(2 \pi i(q ; q)_{\infty}\right)$. A simple calculation shows that the residue of the function $\left(e^{3 \pi i z^{2} / \tau} / \sqrt{-i \tau}\right) g\left(-\frac{z}{\tau} ;-\frac{1}{\tau}\right)$ at $-\frac{1}{2} \tau-\frac{1}{2}$ is $q^{1 / 3} /\left(2 \pi i(q ; q)_{\infty}\right)$. Using these results and the two functional equations satisfied by $G(z ; \tau)$, we easily verify that $G(z ; \tau)$ is a holomorphic function of $z$.

Now we discuss the left-hand side of (33). Let $H(z ; \tau)$ denote the left-hand side of (33). Then, by the definition of $H(z ; \tau)$, we get

$$
\begin{aligned}
H(z+1 ; \tau)-H(z ; \tau)= & \frac{1}{\sqrt{3}} \int_{-\infty}^{\infty} \frac{e^{\frac{\pi i \tau x^{2}}{3}-2 \pi z x}\left(e^{-2 \pi x}-1\right)}{e^{\frac{2 \pi x}{3}}+1+e^{-\frac{2 \pi x}{3}}} d x \\
= & \frac{e^{\frac{\pi i(3 z+2)^{2}}{3 \tau}}}{\sqrt{3}} \int_{-\infty}^{\infty} e^{\frac{\pi i \tau}{3}\left\{x+\frac{i}{\tau}(3 z+2)\right\}^{2}} d x \\
& -\frac{e^{\frac{\pi i(3 z+1)^{2}}{3 \tau}}}{\sqrt{3}} \int_{-\infty}^{\infty} e^{\frac{\pi i \tau}{3}\left\{x+\frac{i}{\tau}(3 z+1)\right\}^{2}} d x .
\end{aligned}
$$

Recall that $\tau=i n$. If $z$ is a real number then we easily show that each of two integrals equals $\sqrt{3 i / \tau}$. Assume that $z$ is a complex number such that $\Im(z) \neq 0$. We consider the first integral on the right-hand side of the equation above.

$$
\int_{-\infty}^{\infty} e^{\frac{\pi i \tau}{3}\left\{x+\frac{i}{\tau}(3 z+2)\right\}^{2}} d x=\int_{-\infty+\frac{i}{\tau}(3 z+2)}^{\infty+\frac{i}{\tau}(3 z+2)} e^{\frac{\pi i \tau}{3} x^{2}} d x
$$

For a positive number $t$, we consider the integral

$$
\int_{\gamma} e^{\frac{\pi i \tau}{3} x^{2}} d x
$$

taken around the rectangle $\gamma$ whose vertices are at the points $\pm t$ and $\pm t+\frac{i}{\tau}(3 z+2)$. By Cauchy's residue theorem, we easily get that the integral above equals 0 . We first evaluate

$$
\int_{-t}^{-t+\frac{i}{\tau}(3 z+2)} e^{\frac{\pi i \tau}{3} x^{2}} d x .
$$

Let $z=a+b i$ where $a$ and $b$ are real and $b \neq 0$. We only need to consider three cases: $(3 a+2) / n=0,(3 a+2) / n>0$, and $(3 a+2) / n<0$. If $(3 a+2) / n=0$, then

$$
\left|\int_{-t}^{-t+\frac{i}{\tau}(3 z+2)} e^{\frac{\pi i \tau}{3} x^{2}} d x\right| \leq \frac{3 b}{n} e^{-\frac{\pi n}{3} t^{2}+\frac{3 \pi b^{2}}{n}} .
$$


Thus, $\int_{-t}^{-t+\frac{i}{\tau}(3 z+2)} e^{\frac{\pi i \tau}{3} x^{2}} d x$ tends to 0 as $t$ tends to $\infty$. If $(3 a+2) / n$ is positive (or negative) then there is a real number $c$ such that $-t<c<-t+(3 a+2) / n$ (or $-t+(3 a+2) / n<c<-t)$ and

$$
\left|\int_{-t}^{-t+\frac{i}{\tau}(3 z+2)} e^{\frac{\pi i \tau}{3} x^{2}} d x\right| \leq \frac{\sqrt{(3 a+2)^{2}+9 b^{2}}}{n} e^{-\frac{\pi n}{3} c^{2}+\frac{3 \pi b^{2}}{n}} .
$$

Thus, the integral $\int_{-t}^{-t+\frac{i}{\tau}(3 z+2)} e^{\frac{\pi i \tau}{3} x^{2}} d x$ tends to 0 as $t$ tends to $\infty$. Similarly, $\int_{t}^{t+\frac{i}{\tau}(3 z+2)} e^{\frac{\pi i \tau}{3} x^{2}} d x$ tends to 0 as $t$ tends to $\infty$. Therefore, we see that

$$
\int_{-\infty+\frac{i}{\tau}(3 z+2)}^{\infty+\frac{i}{\tau}(3 z+2)} e^{\frac{\pi i \tau}{3} x^{2}} d x=\int_{-\infty}^{\infty} e^{\frac{\pi i \tau}{3} x^{2}} d x=\sqrt{\frac{3 i}{\tau}} .
$$

After a simple calculation, we obtain

$$
H(z+1 ; \tau)-H(z ; \tau)=\frac{e^{\frac{3 \pi i(z+1)^{2}}{\tau}}}{\sqrt{-i \tau}}\left(e^{-\frac{5 \pi i}{3 \tau}-\frac{2 \pi i z}{\tau}}-e^{-\frac{8 \pi i}{3 \tau}-\frac{4 \pi i z}{\tau}}\right) .
$$

Next, we discuss $e^{-3 \pi i \tau-6 \pi i z} H(z+\tau ; \tau)-H(z ; \tau)$. After simple calculations and integration by substitution, we get

$$
\begin{aligned}
& e^{-3 \pi i \tau-6 \pi i z} H(z+\tau ; \tau)-H(z ; \tau) \\
& \quad=\frac{1}{\sqrt{3}} \int_{-\infty+3 i}^{\infty+3 i} \frac{e^{\frac{\pi i \tau x^{2}}{3}}-2 \pi z x}{e^{\frac{2 \pi x}{3}}+1+e^{-\frac{2 \pi x}{3}}} d x-\frac{1}{\sqrt{3}} \int_{-\infty}^{\infty} \frac{e^{\frac{\pi i \tau x^{2}}{3}}-2 \pi z x}{e^{\frac{2 \pi x}{3}}+1+e^{-\frac{2 \pi x}{3}}} d x .
\end{aligned}
$$

For a positive number $s$, we consider the integral

$$
\int \frac{e^{\frac{\pi i \tau x^{2}}{3}-2 \pi z x}}{e^{\frac{2 \pi x}{3}}+1+e^{-\frac{2 \pi x}{3}}} d x
$$

taken around the rectangle $\delta$ whose vertices are at the points $\pm s$ and $\pm s+3 i$. By Cauchy's residue theorem, after some elementary algebra, we find that the integral above equals $\sqrt{3} e^{-\frac{\pi i \tau}{3}-2 \pi i z}\left(1-e^{-\pi i \tau-2 \pi i z}\right)$. We first evaluate

$$
\int_{s}^{s+3 i} \frac{e^{\frac{\pi i \tau x^{2}}{3}}-2 \pi z x}{e^{\frac{2 \pi x}{3}}+1+e^{-\frac{2 \pi x}{3}}} d x .
$$

We again recall that $\tau=i n$. Since, for any $y$ such that $0<y<3$,

$$
\left|\frac{e^{\frac{\pi i \tau(s+y i)^{2}}{3}}-2 \pi z(s+y i)}{e^{\frac{2 \pi(s+y i)}{3}}+1+e^{-\frac{2 \pi(s+y i)}{3}}}\right|
$$


tends to 0 as $s$ tends to $\infty$, we easily find that the integral above tends to 0 as $s$ tends to $\infty$. Similarly, we deduce that

$$
\int_{-s}^{-s+3 i} \frac{e^{\frac{\pi i \tau x^{2}}{3}}-2 \pi z x}{e^{\frac{2 \pi x}{3}}+1+e^{-\frac{2 \pi x}{3}}} d x
$$

tends to 0 as $s$ tends to $\infty$. Therefore, we obtain

$$
e^{-3 \pi i \tau-6 \pi i z} H(z+\tau ; \tau)-H(z ; \tau)=e^{-\frac{\pi i \tau}{3}-2 \pi i z}\left(1-e^{-\pi i \tau-2 \pi i z}\right) .
$$

After some elementary algebra and elementary manipulation, we find that $H(z ; \tau)$ also satisfies the same functional equations as $G(z ; \tau)$ and is a holomorphic function of $z$.

Let $F(z ; \tau):=H(z ; \tau)-G(z ; \tau)$. Then, by the functional equations satisfied by $H$ and $G$, we obtain

$$
F(z+1 ; \tau)=F(z ; \tau) \text { and } F(z+\tau ; \tau)=e^{3 \pi i \tau+6 \pi i z} F(z ; \tau) .
$$

Let $T$ be a set of complex numbers such that for any $t \in T, 0 \leq \Re(t) \leq 1$ and $0 \leq \Im(t) \leq n$. Since $T$ is a compact set, $F(z ; \tau)$ is bounded on $T$. For any $t^{\prime} \in \mathbb{C} \backslash T$, there are two integers $k$ and $l$ and a complex number $t$ such that $t \in T$ and $t^{\prime}=t+k \tau+l$. Thus, using repeatedly the functional equations satisfied by $F(z ; \tau)$,

$$
F\left(t^{\prime}\right)=F(t+k \tau+l)=F(t+k \tau)=e^{-3 \pi n k^{2}+6 \pi i t k} F(t) .
$$

Hence,

$$
\left|F\left(t^{\prime}\right)\right|=e^{-3 \pi n k^{2}-6 \pi k \Im t}|F(t)| \leq e^{-3 \pi n\left\{(|k|-1)^{2}-1\right\}}|F(t)| .
$$

So, we are able to say that $F$ is bounded on $\mathbb{C}$. Therefore, by Liouville's theorem, $F(z ; \tau)$ is a constant. In (38), $F$ tends to 0 as $k$ tends to $\infty$. This implies that $F(z ; \tau)=0$. Finally, we have proved (33).

\section{The proof of the second identity}

Proof of Theorem 2. Employing (6) with a moderate modification, we derive (39)

$$
\sum_{m=1}^{\infty} \frac{q^{\frac{2 m(m-1)}{3}}}{\left(-e^{\pi t} q^{\frac{1}{3}} ; q^{\frac{2}{3}}\right)_{m}\left(-e^{-\pi t} q^{\frac{1}{3}} ; q^{\frac{2}{3}}\right)_{m}}=\frac{1}{\left(q^{\frac{2}{3}} ; q^{\frac{2}{3}}\right)_{\infty}} \sum_{m=-\infty}^{\infty} \frac{(-1)^{m} q^{m(m+1)}}{1+q^{\frac{2}{3} m+\frac{1}{3}} e^{\pi t}},
$$

$$
\sum_{m=1}^{\infty} \frac{q_{1}^{6 m(m-1)}}{\left(-e^{\frac{\pi i t}{n}} q_{1}^{3} ; q_{1}^{6}\right)_{m}\left(-e^{-\frac{\pi i t}{n}} q_{1}^{3} ; q_{1}^{6}\right)_{m}}=\frac{1}{\left(q_{1}^{6} ; q_{1}^{6}\right)_{\infty}} \sum_{m=-\infty}^{\infty} \frac{(-1)^{m} q_{1}^{9 m^{2}+9 m}}{1+q_{1}^{6 m+3} e^{\frac{\pi i t}{n}}} .
$$


By a straightforward calculation, we easily find that

$$
\begin{aligned}
\sum_{m=-\infty}^{\infty} \frac{(-1)^{m} q^{m(m+1)}}{1+q^{\frac{2}{3} m+\frac{1}{3}} e^{\pi t}} & \\
= & \sum_{m=1}^{\infty} \frac{(-1)^{m+1} q^{m(m-1)}}{1+q^{\frac{2}{3} m-\frac{1}{3}} e^{\pi t}}+\sum_{m=1}^{\infty} \frac{(-1)^{m} q^{m(m-1)}}{1+q^{-\frac{2}{3} m+\frac{1}{3}} e^{\pi t}} \\
& =\sum_{m=1}^{\infty} \frac{(-1)^{m+1} q^{m(m-1)}}{1+q^{\frac{2}{3} m-\frac{1}{3}} e^{\pi t}}+\sum_{m=1}^{\infty} \frac{(-1)^{m} q^{m(m-1)}\left(q^{\frac{2}{3} m-\frac{1}{3}} e^{-\pi t}+1-1\right)}{1+q^{\frac{2}{3} m-\frac{1}{3}} e^{-\pi t}} \\
& =\sum_{m=1}^{\infty} \frac{(-1)^{m+1} q^{m(m-1)}}{1+q^{\frac{2}{3} m-\frac{1}{3}} e^{\pi t}}+\sum_{m=1}^{\infty} \frac{(-1)^{m+1} q^{m(m-1)}}{1+q^{\frac{2}{3} m-\frac{1}{3}} e^{-\pi t}}-\sum_{m=1}^{\infty}(-1)^{m+1} q^{m(m-1)} .
\end{aligned}
$$

Similarly, we obtain

$$
\begin{aligned}
\sum_{m=-\infty}^{\infty} \frac{(-1)^{m} q_{1}^{9 m^{2}+9 m}}{1+q_{1}^{6 m+3} e^{\frac{\pi i t}{n}}} & =\sum_{m=1}^{\infty} \frac{(-1)^{m+1} q_{1}^{9 m^{2}-9 m}}{1+q_{1}^{6 m-3} e^{\frac{\pi i t}{n}}} \\
& +\sum_{m=1}^{\infty} \frac{(-1)^{m+1} q_{1}^{9 m^{2}-9 m}}{1+q_{1}^{6 m-3} e^{-\frac{\pi i t}{n}}}-\sum_{m=1}^{\infty}(-1)^{m+1} q_{1}^{9 m^{2}-9 m}
\end{aligned}
$$

We previously derived that

$$
\left(q^{\frac{2}{3}} ; q^{\frac{2}{3}}\right)_{\infty}=\frac{i(1-\omega)}{\sqrt{3 n}} q^{-\frac{1}{36}} q_{1}^{\frac{1}{4}} e^{-\frac{\pi i}{3}}\left(q_{1}^{6} ; q_{1}^{6}\right)_{\infty} \quad \text { and } \quad i(1-\omega) e^{-\frac{\pi i}{3}}=\sqrt{3} .
$$

Thus, using these, we obtain

$$
\frac{q^{-\frac{1}{36}}}{\left(q^{\frac{2}{3}} ; q^{\frac{2}{3}}\right)_{\infty}} \frac{q_{1}^{\frac{9}{4}}}{n}=\frac{q_{1}^{2}}{\sqrt{n}\left(q_{1}^{6} ; q_{1}^{6}\right)_{\infty}} .
$$

Combining equations (39)-(43) completes the proof.

\section{Modular transformations derived from Ramanujan's identity}

In this section, we derive modular transformations for third-order mock theta functions from Theorem 1.

Ramanujan's third-order mock theta functions are defined by

$$
\begin{gathered}
f(q)=\sum_{m=0}^{\infty} \frac{q^{m^{2}}}{(-q ; q)_{m}^{2}}, \quad \phi(q)=\sum_{m=0}^{\infty} \frac{q^{m^{2}}}{\left(-q^{2} ; q^{2}\right)_{m}}, \quad \psi(q)=\sum_{m=1}^{\infty} \frac{q^{m^{2}}}{\left(q ; q^{2}\right)_{m}} \\
\chi(q)=\sum_{m=0}^{\infty} \frac{q^{m^{2}}}{(-\omega q ; q)_{m}\left(-\omega^{2} ; q\right)_{m}}
\end{gathered}
$$


Watson's third-order mock theta functions are defined by

$$
\begin{gathered}
\omega(q)=\sum_{m=1}^{\infty} \frac{q^{2 m(m-1)}}{\left(q ; q^{2}\right)_{m}^{2}}, \quad v(q)=\sum_{m=1}^{\infty} \frac{q^{m(m-1)}}{\left(-q ; q^{2}\right)_{m}}, \\
\rho(q)=\sum_{m=1}^{\infty} \frac{q^{2 m(m-1)}}{\left(\omega q ; q^{2}\right)_{m}\left(\omega^{2} q ; q^{2}\right)_{m}} .
\end{gathered}
$$

Gordon and McIntosh's third-order mock theta functions are defined by

$$
\xi(q)=1+2 \sum_{m=1}^{\infty} \frac{q^{6 m(m-1)}}{\left(q ; q^{6}\right)_{m}\left(q^{5} ; q^{6}\right)_{m}}, \quad \sigma(q)=\sum_{m=1}^{\infty} \frac{q^{3 m(m-1)}}{\left(-q ; q^{3}\right)_{m}\left(-q^{2} ; q^{3}\right)_{m}} .
$$

To apply Theorem 1 directly to these functions, we first need new representations for Ramanujan's mock theta functions. With his formula for basic hypergeometric series, Watson [1936] gave new representations for $\phi(q)$ and $\psi(q)$, namely,

$$
\begin{aligned}
& \phi(q)=\frac{1}{(q ; q)_{\infty}}\left(1+2 \sum_{m=1}^{\infty} \frac{(-1)^{m}\left(1+q^{m}\right) q^{m(3 m+1) / 2}}{1+q^{2 m}}\right) \\
& \psi(q)=\frac{1}{\left(q^{4} ; q^{4}\right)_{\infty}} \sum_{m=-\infty}^{\infty} \frac{(-1)^{m} q^{6 m(m+1)+1}}{1-q^{4 m+1}}
\end{aligned}
$$

Then, using the definition of $f(q)$ and applying (5) with $z$ replaced by -1 , we deduce that

$$
f(q)=2-2 \sum_{m=1}^{\infty} \frac{q^{m(m-1)}}{(-1 ; q)_{m}(-q ; q)_{m}} .
$$

Using the definition of $\phi(q)$ and applying (5) with $z$ replaced by $i$ and $-i$, respectively, we obtain

$$
\begin{aligned}
\phi(q) & =(1-i)\left(1+i \sum_{m=1}^{\infty} \frac{q^{m(m-1)}}{(i ; q)_{m}(-i q ; q)_{m}}\right) \\
& =(1+i)\left(1-i \sum_{m=1}^{\infty} \frac{q^{m(m-1)}}{(-i ; q)_{m}(i q ; q)_{m}}\right) .
\end{aligned}
$$

Using (45) and applying (6) with $q$ and $z$ replaced by $q^{4}$ and $q$, respectively, we deduce that

$$
\psi(q)=q \sum_{m=1}^{\infty} \frac{q^{4 m(m-1)}}{\left(q ; q^{4}\right)_{m}\left(q^{3} ; q^{4}\right)_{m}} .
$$

Using the definition of $\chi(q)$ and applying (5) with $z$ replaced by $-\omega$ and $-\omega^{2}$, respectively, we have 


$$
\begin{aligned}
\chi(q) & =(1+\omega)\left(1-\omega \sum_{m=1}^{\infty} \frac{q^{m(m-1)}}{(-\omega ; q)_{m}\left(-\omega^{2} q ; q\right)_{m}}\right) \\
& =\left(1+\omega^{2}\right)\left(1-\omega^{2} \sum_{m=1}^{\infty} \frac{q^{m(m-1)}}{\left(-\omega^{2} ; q\right)_{m}(-\omega q ; q)_{m}}\right) .
\end{aligned}
$$

We are now ready to derive modular transformations from Theorem 1 . We record here the ones which are derived directly from Theorem 1 and expressed in terms of Mordell integrals and third-order mock theta functions. Similar modular transformations can be found in [Gordon and McIntosh 2012].

Using Theorem 1 with $t$ replaced by $n-\frac{i}{2}$ and $n+\frac{i}{2}$, respectively, we obtain

$$
\begin{aligned}
& \frac{2}{\sqrt{3}} \int_{0}^{\infty} \frac{e^{-\frac{\pi n x^{2}}{3}} \cos \pi\left(n-\frac{i}{2}\right) x}{e^{\frac{2 \pi x}{3}}+1+e^{-\frac{2 \pi x}{3}}} d x=q^{\frac{2}{9}}\left(\frac{\phi\left(q^{\frac{2}{3}}\right)}{1+i}+i\right)-\frac{\sqrt{2} q^{\frac{1}{4}} q_{1}^{-\frac{1}{16}}}{(1+i) \sqrt{n}} \psi\left(q_{1}^{\frac{3}{2}}\right), \\
& \frac{2}{\sqrt{3}} \int_{0}^{\infty} \frac{e^{-\frac{\pi n x^{2}}{3}} \cos \pi\left(n+\frac{i}{2}\right) x}{e^{\frac{2 \pi x}{3}}+1+e^{-\frac{2 \pi x}{3}}} d x=q^{\frac{2}{9}}\left(\frac{\phi\left(q^{\frac{2}{3}}\right)}{1-i}-i\right)-\frac{\sqrt{2} q^{\frac{1}{4}} q_{1}^{-\frac{1}{16}}}{(1-i) \sqrt{n}} \psi\left(q_{1}^{\frac{3}{2}}\right) .
\end{aligned}
$$

Adding the two results above and calculating straightforwardly, we have

$$
\frac{4}{\sqrt{3}} \int_{0}^{\infty} \frac{e^{-\frac{\pi n x^{2}}{3}} \cos \pi n x \cosh \frac{\pi x}{2}}{e^{\frac{2 \pi x}{3}}+1+e^{-\frac{2 \pi x}{3}}} d x=q^{\frac{2}{9}} \phi\left(q^{\frac{2}{3}}\right)-\sqrt{\frac{2}{n}} q^{\frac{1}{4}} q_{1}^{-\frac{1}{16}} \psi\left(q_{1}^{\frac{3}{2}}\right)
$$

Using Theorem 1 with $t$ replaced by $\frac{n}{2}-i$ and $-\frac{n}{2}-i$, respectively, we obtain

$$
\begin{aligned}
& \frac{2}{\sqrt{3}} \int_{0}^{\infty} \frac{e^{-\frac{\pi n x^{2}}{3}} \cos \pi\left(\frac{n}{2}-i\right) x}{e^{\frac{2 \pi x}{3}}+1+e^{-\frac{2 \pi x}{3}}} d x \\
& =q^{\frac{1}{18}} \psi\left(q^{\frac{1}{6}}\right)-\frac{q^{\frac{1}{16}} q_{1}^{-\frac{1}{4}}}{\sqrt{2 n}} \phi\left(q_{1}^{6}\right)+\frac{q^{\frac{1}{16}} q_{1}^{-\frac{1}{4}} e^{\pi i / 4}}{\sqrt{n}}, \\
& \frac{2}{\sqrt{3}} \int_{0}^{\infty} \frac{e^{-\frac{\pi n x^{2}}{3}} \cos \pi\left(\frac{n}{2}+i\right) x}{e^{\frac{2 \pi x}{3}}+1+e^{-\frac{2 \pi x}{3}}} d x \\
& =q^{\frac{1}{18}} \psi\left(q^{\frac{1}{6}}\right)-\frac{q^{\frac{1}{16}} q_{1}^{-\frac{1}{4}}}{\sqrt{2 n}} \phi\left(q_{1}^{6}\right)+\frac{q^{\frac{1}{16}} q_{1}^{-\frac{1}{4}} e^{-\pi i / 4}}{\sqrt{n}} .
\end{aligned}
$$

Adding the two results above and calculating straightforwardly, we find that

$$
\frac{2}{\sqrt{3}} \int_{0}^{\infty} \frac{e^{-\frac{\pi n x^{2}}{3}} \cos \frac{\pi n x}{2} \cosh \pi x}{e^{\frac{2 \pi x}{3}}+1+e^{-\frac{2 \pi x}{3}}} d x=q^{\frac{1}{18}} \psi\left(q^{\frac{1}{6}}\right)-\frac{q^{\frac{1}{16}} q_{1}^{-\frac{1}{4}}}{\sqrt{2 n}}\left(\phi\left(q_{1}^{6}\right)-1\right)
$$


Using Theorem 1 with $t$ replaced by $n+\frac{2}{3} i$ and $-n+\frac{2}{3} i$, respectively, we obtain

$$
\begin{aligned}
& \frac{2}{\sqrt{3}} \int_{0}^{\infty} \frac{e^{-\frac{\pi n x^{2}}{3}} \cos \pi\left(n+\frac{2}{3} i\right) x}{e^{\frac{2 \pi x}{3}}+1+e^{-\frac{2 \pi x}{3}}} d x=q^{\frac{2}{9}}\left(\omega^{2}+\chi\left(q^{\frac{1}{3}}\right)\right)-\frac{q^{\frac{1}{4}} q_{1}}{2 \sqrt{n}}\left(\xi\left(q_{1}\right)-1\right), \\
& \frac{2}{\sqrt{3}} \int_{0}^{\infty} \frac{e^{-\frac{\pi n x^{2}}{3}} \cos \pi\left(n-\frac{2}{3} i\right) x}{e^{\frac{2 \pi x}{3}}+1+e^{-\frac{2 \pi x}{3}}} d x=q^{\frac{2}{9}}\left(\omega+\chi\left(q^{\frac{1}{3}}\right)\right)-\frac{q^{\frac{1}{4}} q_{1}}{2 \sqrt{n}}\left(\xi\left(q_{1}\right)-1\right) .
\end{aligned}
$$

Adding the two results above and calculating straightforwardly, we find that

$$
\frac{4}{\sqrt{3}} \int_{0}^{\infty} \frac{e^{-\frac{\pi n x^{2}}{3}} \cos \pi n x \cosh \frac{2 \pi x}{3}}{e^{\frac{2 \pi x}{3}}+1+e^{-\frac{2 \pi x}{3}}} d x=q^{\frac{2}{9}}\left(2 \chi\left(q^{\frac{1}{3}}\right)-1\right)-\frac{q^{\frac{1}{4}} q_{1}}{\sqrt{n}}\left(\xi\left(q_{1}\right)-1\right) .
$$

Using Theorem 1 with $t$ replaced by $n, \frac{n}{2}, i, 0, \frac{i}{2},-\frac{i}{3}, \frac{2 i}{3}$, and $\frac{n}{3}$, respectively, we obtain,

$$
\begin{aligned}
& \frac{2}{\sqrt{3}} \int_{0}^{\infty} \frac{e^{-\frac{\pi n x^{2}}{3}} \cos \pi n x}{e^{\frac{2 \pi x}{3}}+1+e^{-\frac{2 \pi x}{3}}} d x=q^{\frac{2}{9}}\left(1-\frac{1}{2} f(q)\right)+\frac{q^{\frac{1}{4}} q_{1}^{2}}{\sqrt{n}} \omega\left(q_{1}^{3}\right), \\
& \frac{2}{\sqrt{3}} \int_{0}^{\infty} \frac{e^{-\frac{\pi n x^{2}}{3}} \cos \frac{\pi n x}{2}}{e^{\frac{2 \pi x}{3}}+1+e^{-\frac{2 \pi x}{3}}} d x=-q^{\frac{1}{18}} \psi\left(-q^{\frac{1}{6}}\right)+\frac{q^{\frac{1}{16}} q_{1}^{2}}{\sqrt{n}} v\left(q_{1}^{6}\right)
\end{aligned}
$$$$
\frac{2}{\sqrt{3}} \int_{0}^{\infty} \frac{e^{-\frac{\pi n x^{2}}{3}} \cosh \pi x}{e^{\frac{2 \pi x}{3}}+1+e^{-\frac{2 \pi x}{3}}} d x=q^{\frac{2}{9}} \omega\left(q^{\frac{1}{3}}\right)+\frac{q_{1}^{-\frac{1}{4}}}{\sqrt{n}}\left(1-\frac{1}{2} f\left(q_{1}^{6}\right)\right),
$$

$$
\frac{2}{\sqrt{3}} \int_{0}^{\infty} \frac{e^{-\frac{\pi n x^{2}}{3}}}{e^{\frac{2 \pi x}{3}}+1+e^{-\frac{2 \pi x}{3}}} d x=q^{\frac{2}{9}} \omega\left(-q^{\frac{1}{3}}\right)+\frac{q_{1}^{2}}{\sqrt{n}} \omega\left(-q_{1}^{3}\right),
$$

$$
\begin{aligned}
& \frac{2}{\sqrt{3}} \int_{0}^{\infty} \frac{e^{-\frac{\pi n x^{2}}{3}} \cosh \frac{\pi x}{2}}{e^{\frac{2 \pi x}{3}}+1+e^{-\frac{2 \pi x}{3}}} d x=q^{\frac{2}{9}} v\left(q^{\frac{2}{3}}\right)-\frac{q_{1}^{-\frac{1}{16}}}{\sqrt{n}} \psi\left(-q_{1}^{\frac{3}{2}}\right), \\
& \frac{2}{\sqrt{3}} \int_{0}^{\infty} \frac{e^{-\frac{\pi n x^{2}}{3}} \cosh \frac{\pi x}{3}}{e^{\frac{2 \pi x}{3}}+1+e^{-\frac{2 \pi x}{3}}} d x=q^{\frac{2}{9}} \rho\left(q^{\frac{1}{3}}\right)+\frac{q_{1}^{\frac{7}{4}}}{\sqrt{n}} \sigma\left(q_{1}^{2}\right),
\end{aligned}
$$$$
\frac{2}{\sqrt{3}} \int_{0}^{\infty} \frac{e^{-\frac{\pi n x^{2}}{3}} \cosh \frac{2 \pi x}{3}}{e^{\frac{2 \pi x}{3}}+1+e^{-\frac{2 \pi x}{3}}} d x=q^{\frac{2}{9}} \rho\left(-q^{\frac{1}{3}}\right)+\frac{q_{1}}{2 \sqrt{n}}\left(\xi\left(-q_{1}\right)-1\right),
$$$$
\frac{2}{\sqrt{3}} \int_{0}^{\infty} \frac{e^{-\frac{\pi n x^{2}}{3}} \cos \frac{\pi n x}{3}}{e^{\frac{2 \pi x}{3}}+1+e^{-\frac{2 \pi x}{3}}} d x=q^{\frac{2}{9}} \sigma\left(q^{\frac{2}{9}}\right)+\frac{q^{\frac{1}{36}} q_{1}^{2}}{\sqrt{n}} \rho\left(q_{1}^{3}\right) .
$$

Here, using (46)-(50), we give evaluations for specific Mordell integrals and new representations for Ramanujan's third-order mock theta functions $\phi, \psi$, and $\omega$. 
Replacing $n$ by $\frac{1}{2}$ in (46), we obtain

$$
\frac{4}{\sqrt{3}} \int_{0}^{\infty} \frac{e^{-\frac{\pi x^{2}}{6}} \cos \frac{\pi x}{2} \cosh \frac{\pi x}{2}}{e^{\frac{2 \pi x}{3}}+1+e^{-\frac{2 \pi x}{3}}} d x=e^{-\frac{\pi}{3}}\left\{\phi\left(e^{-\pi}\right)-2 q \psi\left(e^{-\pi}\right)\right\} .
$$

Replacing $n$ by 2 in (47) and multiplying 2 to both sides of (47), we find

$$
\frac{4}{\sqrt{3}} \int_{0}^{\infty} \frac{e^{-\frac{2 \pi x^{2}}{3}} \cos \pi x \cosh \pi x}{e^{\frac{2 \pi x}{3}}+1+e^{-\frac{2 \pi x}{3}}} d x=e^{-\frac{\pi}{3}}\left\{2 q \psi\left(e^{-\pi}\right)-\phi\left(e^{-\pi}\right)+1\right\} .
$$

Adding (51) and (52), we obtain

$$
\frac{4}{\sqrt{3}}\left(\int_{0}^{\infty} \frac{e^{-\frac{\pi x^{2}}{6}} \cos \frac{\pi x}{2} \cosh \frac{\pi x}{2}}{e^{\frac{2 \pi x}{3}}+1+e^{-\frac{2 \pi x}{3}}} d x+\int_{0}^{\infty} \frac{e^{-\frac{2 \pi x^{2}}{3}} \cos \pi x \cosh \pi x}{e^{\frac{2 \pi x}{3}}+1+e^{-\frac{2 \pi x}{3}}} d x\right)=e^{-\frac{\pi}{3}} .
$$

Replacing $n$ by 2 in (48) and replacing $n$ by $\frac{1}{2}$ in (50), we obtain

$$
\begin{aligned}
& \frac{2}{\sqrt{3}} \int_{0}^{\infty} \frac{e^{-\frac{2 \pi x^{2}}{3}} \cos \pi x}{e^{\frac{2 \pi x}{3}}+1+e^{-\frac{2 \pi x}{3}}} d x=-e^{-\frac{\pi}{3}} \psi\left(-e^{-\pi}\right)+\frac{e^{-\frac{17}{24} \pi}}{\sqrt{2}} v\left(e^{-\pi}\right) \\
& \frac{2}{\sqrt{3}} \int_{0}^{\infty} \frac{e^{-\frac{\pi x^{2}}{6}} \cosh \frac{\pi x}{2}}{e^{\frac{2 \pi x}{3}}+1+e^{-\frac{2 \pi x}{3}}} d x=e^{-\frac{\pi}{3}} v\left(e^{-\pi}\right)-\sqrt{2} e^{\frac{\pi}{24}} \psi\left(-e^{-\pi}\right) .
\end{aligned}
$$

Comparing (53) and (54), we have

$$
\int_{0}^{\infty} \frac{e^{-\frac{2 \pi x^{2}}{3}} \cos \pi x}{e^{\frac{2 \pi x}{3}}+1+e^{-\frac{2 \pi x}{3}}} d x=\frac{e^{-\frac{3}{8} \pi}}{\sqrt{2}} \int_{0}^{\infty} \frac{e^{-\frac{\pi x^{2}}{6}} \cosh \frac{\pi x}{2}}{e^{\frac{2 \pi x}{3}}+1+e^{-\frac{2 \pi x}{3}}} d x .
$$

Ramanujan [1988] recorded

$$
\phi(q)+2 \psi(q)=\frac{(-q ;-q)_{\infty}}{(q ;-q)_{\infty}^{2}},
$$

which was proved by Watson [1936]. The right-hand side of (55) can be expressed in terms of theta functions. Using (51), (52), and (55), we obtain new representations for $\phi$ and $\psi$ which are

$$
\begin{aligned}
& \phi\left(e^{-\pi}\right)=\frac{2}{\sqrt{3}} e^{\frac{\pi}{3}} \int_{0}^{\infty} \frac{e^{-\frac{\pi x^{2}}{6}} \cos \frac{\pi x}{2} \cosh \frac{\pi x}{2}}{e^{\frac{2 \pi x}{3}}+1+e^{-\frac{2 \pi x}{3}}} d x+\frac{1}{2} \frac{\left(-e^{-\pi} ;-e^{-\pi}\right)_{\infty}}{\left(e^{-\pi} ;-e^{-\pi}\right)_{\infty}^{2}} \\
& \psi\left(e^{-\pi}\right)=-\frac{1}{\sqrt{3}} e^{\frac{\pi}{3}} \int_{0}^{\infty} \frac{e^{-\frac{2 \pi x^{2}}{3}} \cos \pi x \cosh \pi x}{e^{\frac{2 \pi x}{3}}+1+e^{-\frac{2 \pi x}{3}}} d x+\frac{1}{4} \frac{\left(-e^{-\pi} ;-e^{-\pi}\right)_{\infty}}{\left(e^{-\pi} ;-e^{-\pi}\right)_{\infty}^{2}} .
\end{aligned}
$$

Replacing $n$ by 1 in (49), we obtain a new representation for $\omega$, namely,

$$
\omega\left(-e^{-\pi}\right)=\frac{1}{\sqrt{3}} e^{\frac{2 \pi}{3}} \int_{0}^{\infty} \frac{e^{-\frac{\pi x^{2}}{3}}}{e^{\frac{2 \pi x}{3}}+1+e^{-\frac{2 \pi x}{3}}} d x .
$$




\section{Acknowledgments}

The author is grateful to Bruce C. Berndt for introducing him to Ramanujan's modular transformations in Ramanujan's Lost Notebook. The author also thanks the referees for their inspiration and valuable comments.

\section{References}

[Andrews 1981] G. E. Andrews, "Mordell integrals and Ramanujan's 'lost' notebook”, pp. 10-48 in Analytic number theory (Philadelphia, 1980), edited by M. I. Knopp, Lecture Notes in Math. 899, Springer, Berlin, 1981. MR 83m:33004 Zbl 0482.33002

[Andrews and Garvan 1989] G. E. Andrews and F. G. Garvan, "Ramanujan's "lost" notebook, VI: The mock theta conjectures", Adv. in Math. 73:2 (1989), 242-255. MR 90d:11115

[Berndt and Rankin 1995] B. C. Berndt and R. A. Rankin, Ramanujan: letters and commentary, History of Mathematics 9, Amer. Math. Soc., Providence, RI, 1995. MR 97c:01034 Zbl 0842.01026 [Chern and Rhoades 2012] B. Chern and R. C. Rhoades, "The Mordell integral and quantum modular forms", preprint, 2012.

[Choi 2002] Y.-S. Choi, "Tenth order mock theta functions in Ramanujan's lost notebook, IV", Trans. Amer. Math. Soc. 354:2 (2002), 705-733. MR 2002k:11022 Zbl 1043.33012

[Choi 2004] Y.-S. Choi, "Generalization of two identities in Ramanujan's lost notebook", Acta Arith. 114:4 (2004), 369-389. MR 2005g:11024 Zbl 1099.11006

[Choi 2011] Y.-S. Choi, "The basic bilateral hypergeometric series and the mock theta functions", Ramanujan J. 24:3 (2011), 345-386. MR 2012e:33042 Zbl 1225.33019

[Garvan 1988] F. G. Garvan, "New combinatorial interpretations of Ramanujan's partition congruences mod 5, 7 and 11”, Trans. Amer. Math. Soc. 305:1 (1988), 47-77. MR 89b:11081 Zbl 0641.10009

[Gordon and McIntosh 2003] B. Gordon and R. J. McIntosh, "Modular transformations of Ramanujan's fifth and seventh order mock theta functions", Ramanujan J. 7:1-3 (2003), 193-222. MR 2005a:11020 Zbl 1031.11008

[Gordon and McIntosh 2012] B. Gordon and R. J. McIntosh, "A survey of classical mock theta functions", pp. 95-144 in Partitions, q-series, and modular forms (Gainesville, FL, 2008), edited by K. Alladi and F. Garvan, Dev. Math. 23, Springer, New York, 2012. MR 3051186 Zbl 1246.33006

[Hickerson 1988] D. Hickerson, "A proof of the mock theta conjectures", Invent. Math. 94:3 (1988), 639-660. MR 90f:11028a Zbl 0661.10059

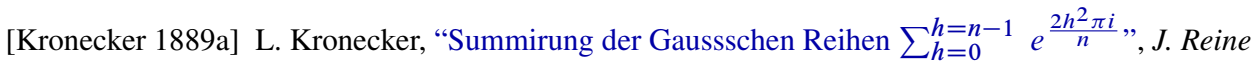
Angew. Math. 105 (1889), 267-268. JFM 21.0251.01

[Kronecker 1889b] L. Kronecker, "Bemerkungen über die Darstellung von Reihen durch Integrale", J. Reine Angew. Math. 105 (1889), 345-351. JFM 21.0281.01 [Mordell 1920] L. J. Mordell, "The value of the definite integral $\int_{-\infty}^{\infty} a^{a t^{2}+b t} /\left(e^{c t}+d\right) d t$ ", Quart.

[Mordell 1933] L. J. Mordell, "The definite integral $\int^{\infty} e^{a x^{2}+b x} /\left(e^{a x}+d\right) d a$ and the analytic theory of numbers”, Acta Math. 61:1 (1933), 323-360.9MR 1555379 Zbl 0008.05501

[Ramanujan 1988] S. Ramanujan, The lost notebook and other unpublished papers, Narosa, New Delhi, 1988. MR 89j:01078 Zbl 0639.01023

[Siegel 1932] C. L. Siegel, “Über Riemanns Nachlaß zur analytischen Zahlentheorie”, Quell. Stud. Gesch. Math. Astro. Phys. B 2 (1932), 45-80. Zbl 0004.10501 JFM 58.1037.07 
[Watson 1936] G. N. Watson, "The final problem: an account of the mock theta functions", J. London Math. Soc. (2) 11:1 (1936), 55-80. MR 1573993 Zbl 0013.11502 JFM 62.0430.02

[Zwegers 2002] S. Zwegers, Mock theta functions, thesis, Utrecht University, 2002, Available at http://dspace.library.uu.nl/handle/1874/878. Zbl 1194.11058

Received April 9, 2013. Revised August 19, 2013.

Youn-SeO CHOI

The School of Mathematics

Korea Institute FOR AdVANCED STUdy

85 HoEgiro, DongdaEmun-GU

SEOUL 130-722

SOUTH KOREA

y-choi2@kias.re.kr 


\title{
PACIFIC JOURNAL OF MATHEMATICS
}

\author{
msp.org/pjm
}

Founded in 1951 by E. F. Beckenbach (1906-1982) and F. Wolf (1904-1989)

\section{EDITORS}

Don Blasius (Managing Editor)

Department of Mathematics

University of California

Los Angeles, CA 90095-1555

blasius@math.ucla.edu

\author{
Paul Balmer \\ Department of Mathematics \\ University of California \\ Los Angeles, CA 90095-1555 \\ balmer@math.ucla.edu \\ Robert Finn \\ Department of Mathematics \\ Stanford University \\ Stanford, CA 94305-2125 \\ finn@math.stanford.edu \\ Sorin Popa \\ Department of Mathematics \\ University of California \\ Los Angeles, CA 90095-1555 \\ popa@math.ucla.edu
}

\author{
Vyjayanthi Chari \\ Department of Mathematics \\ University of California \\ Riverside, CA 92521-0135 \\ chari@math.ucr.edu \\ Kefeng Liu \\ Department of Mathematics \\ University of California \\ Los Angeles, CA 90095-1555 \\ liu@math.ucla.edu \\ Jie Qing \\ Department of Mathematics \\ University of California \\ Santa Cruz, CA 95064 \\ qing@ cats.ucsc.edu
}

\section{PRODUCTION}

Silvio Levy, Scientific Editor, production@msp.org

\section{SUPPORTING INSTITUTIONS}

ACADEMIA SINICA, TAIPEI

CALIFORNIA INST. OF TECHNOLOGY

INST. DE MATEMÁTICA PURA E APLICADA

KEIO UNIVERSITY

MATH. SCIENCES RESEARCH INSTITUTE

NEW MEXICO STATE UNIV.

OREGON STATE UNIV.

\author{
STANFORD UNIVERSITY \\ UNIV. OF BRITISH COLUMBIA \\ UNIV. OF CALIFORNIA, BERKELEY \\ UNIV. OF CALIFORNIA, DAVIS \\ UNIV. OF CALIFORNIA, LOS ANGELES \\ UNIV. OF CALIFORNIA, RIVERSIDE \\ UNIV. OF CALIFORNIA, SAN DIEGO \\ UNIV. OF CALIF., SANTA BARBARA
}

\author{
Daryl Cooper \\ Department of Mathematics \\ University of California \\ Santa Barbara, CA 93106-3080 \\ cooper@math.ucsb.edu \\ Jiang-Hua Lu \\ Department of Mathematics \\ The University of Hong Kong \\ Pokfulam Rd., Hong Kong \\ jhlu@maths.hku.hk \\ Paul Yang \\ Department of Mathematics \\ Princeton University \\ Princeton NJ 08544-1000 \\ yang@math.princeton.edu
}

These supporting institutions contribute to the cost of publication of this Journal, but they are not owners or publishers and have no responsibility for its contents or policies.

See inside back cover or msp.org/pjm for submission instructions.

The subscription price for 2014 is US $\$ 410 /$ year for the electronic version, and \$535/year for print and electronic.

Subscriptions, requests for back issues and changes of subscribers address should be sent to Pacific Journal of Mathematics, P.O. Box 4163, Berkeley, CA 94704-0163, U.S.A. The Pacific Journal of Mathematics is indexed by Mathematical Reviews, Zentralblatt MATH, PASCAL CNRS Index, Referativnyi Zhurnal, Current Mathematical Publications and Web of Knowledge (Science Citation Index).

The Pacific Journal of Mathematics (ISSN 0030-8730) at the University of California, c/o Department of Mathematics, 798 Evans Hall \#3840, Berkeley, CA 94720-3840, is published twelve times a year. Periodical rate postage paid at Berkeley, CA 94704, and additional mailing offices. POSTMASTER: send address changes to Pacific Journal of Mathematics, P.O. Box 4163, Berkeley, CA 94704-0163.

PJM peer review and production are managed by EditFLOW ${ }^{\circledR}$ from Mathematical Sciences Publishers.

\section{PUBLISHED BY}

\section{mathematical sciences publishers \\ nonprofit scientific publishing}

http://msp.org/

(C) 2014 Mathematical Sciences Publishers 


\section{PACIFIC JOURNAL OF MATHEMATICS}

Volume $272 \quad$ No. $1 \quad$ November 2014

Nonconcordant links with homology cobordant zero-framed surgery $\quad 1$ manifolds

JAE CHOON CHA and MARK POWELL

Certain self-homotopy equivalences on wedge products of Moore spaces

Ho Won CHOI and KeE Young LeE

Modular transformations involving the Mordell integral in Ramanujan's lost notebook

Youn-SEO CHOI

The $D$-topology for diffeological spaces

J. DANiEl Christensen, Gordon SinNAMON and ENXIN Wu

On the Atkin polynomials

AHMAD EL-Guindy and Mourad E. H. ISMAIL

Evolving convex curves to constant-width ones by a perimeter-preserving flow

LAIYUAN GaO and Shengliang PAN

Hilbert series of certain jet schemes of determinantal varieties

SudhiR R. GHORPADE, Boyan Jonov and B. A. SeTHuraman

On a Liu-Yau type inequality for surfaces

Oussama Hijazi, Sebastián Montiel and Simon Raulot

Nonlinear Euler sums

ISTVÁN MEZŐ

Boundary limits for fractional Poisson $a$-extensions of $L^{p}$ boundary functions 227 in a cone

LEI QIAO and TAO ZHAO

Jacobi-Trudi determinants and characters of minimal affinizations

STEVEN V SAM

Normal families of holomorphic mappings into complex projective space concerning shared hyperplanes

LiU Yang, Caiyun Fang and Xuecheng Pang 